\title{
Scattering of Ion Cyclotron Range of Frequency waves by filaments and ELMs
}

\author{
W. Zhang ${ }^{1, \text { a) }}$, A. Cathey ${ }^{1}$, M. Hoelzl ${ }^{1}$, W. Tierens ${ }^{1}$, V. Bobkov ${ }^{1}$, the ASDEX Upgrade team ${ }^{2}$, \\ the EUROfusion MST1 team ${ }^{3}$ \\ ${ }^{1}$ Max-Planck-Institut für Plasmaphysik, Garching, Germany \\ ${ }^{2}$ see the author list of H. Meyer et al. 2019 Nucl. Fusion 59112014 \\ ${ }^{3}$ see the author list of B. Labit et al 2019 Nucl. Fusion 59086020
}

a) Corresponding author: wei.zhang@ipp.mpg.de

\begin{abstract}
The scattering of Ion Cyclotron Range of Frequency (ICRF) waves by filaments and ELMs has been comprehensively studied via modeling. The influence of filaments on the propagation of ICRF waves is studied by building a 2D COMSOL model which solves the wave equation. It is benchmarked against the Mie scattering theoretical model. Cases either with Perfect Matching Layer boundary condition or with poloidal periodic boundary condition are considered. Parameter scans, including the filament density, the filament radius, the number of filaments as well as the distance between filaments, are performed. Then, the influence of ELMs on the propagation of ICRF waves in realistic geometry is studied by importing density distributions calculated by 3D non-linear MHD code JOREK into the 3D antenna code RAPLICASOL. The evolution of density and wave fields during an ELM is analyzed for cases with a low and high scrape-off layer density, respectively. Both the COMSOL simulation and the realistic simulation with JOREK and RAPLICASOL show that the wave fields and Poynting flux can be significantly and globally modified by the ELM filaments. Due to the mutual influence of many density filaments, radially elongated and poloidally distributed stripe structures of the wave fields develop, leading to poloidally inhomogeneous wave fields. Furthermore, the influence of ELMs on the antenna power and antenna coupling resistance is investigated with experimental results in ASDEX Upgrade.
\end{abstract}




\section{Introduction}

Radio-frequency (RF) heating with waves in the Ion Cyclotron Range of Frequencies (ICRF), Electron Cyclotron Range of Frequencies (ECRF) and Low Hybrid Range of Frequencies (LH) are routinely used to heat thermal plasmas in magnetic confinement fusion devices, such as tokamaks and stellarators. The RF waves are usually excited by antennas or mirror structures at the plasma boundary, and have to propagate through the turbulent scrape-off layer (SOL) before reaching the absorption region in the plasma core. In the SOL, the large and localized density perturbation caused by filaments, especially by Edge Localized Modes (ELMs), can significantly change the local plasma dielectric properties and thus the Stix parameters [1] which determines the propagation of RF waves. A significant wave scattering can be induced by the filaments and it is important to understand this phenomenon. Firstly, the wave scattering can influence the propagation of RF waves and the ultimate RF power absorption in the core plasma. Secondly, the scattered RF waves may cause detrimental effects to the wall. For instance, ECRF wave can cause direct and localized wall hitting and damage in-vessel components [2]. ICRF wave can induce far-field sheath and lead to enhanced impurity generation at the wall [3].

Since the typical filament size ( centimeter) in fusion plasmas is larger than the typical wavelength of ECRF wave (millimeter) and comparable to the typical wavelength of LH wave (centimeter), the observed ECRF and LH wave scattering effect is as prominent as expected. Previously, a lot of interests were concentrated on understanding the scattering of ECRF and LH waves by filaments or turbulence [4-9]. In fact, even the ICRF fast wave whose wavelength is a decimeter to a meter, a length scale much larger than the filament radius, can be significantly scattered, as will be shown in this paper.

The main object of the paper is to understand the scattering of fast wave and its influence on RF heating. The ICRF wave has two branches, the fast wave and slow wave. The fast wave is an X-mode wave, with its phase velocity larger than the speed of light and $E_{\perp} \gg E_{\|}$. It is the one used to heat the main plasma and only propagates at a density larger than the cut-off density, which is in the level of $1 \times 10^{18} \mathrm{~m}^{-3}$ given $\mathrm{f}_{\mathrm{ICRF}}=36.5$

$\mathrm{MHz}, \mathrm{B}_{0}=2 \mathrm{~T}$ and $\mathrm{k}_{\|}=8 \mathrm{~m}^{-1}$. The slow wave is an O-mode wave, with its phase velocity smaller than the speed of light and $E_{\perp} \ll E_{\|}$. It is a parasitically generated and undesirable wave. In the paper, though only the fast wave contribution of the fast wave spectrum launched at the antenna is retained, we consider the fast as well as slow waves in their description of the dielectric tensor and hence inside the plasma. The slow wave generated directly by the antenna is not considered since it is evanescent at a density larger than $4.2 \times 10^{16} \mathrm{~m}^{-3}$ with the above mentioned parameters and only exists in an extremely small layer in the far SOL. In addition, this paper aims at identifying a main reason for why the power reaching the main plasma is critically depending on the perturbed SOL density profile. A further complex investigation of the ultimate fate of this power is not intended in the paper. An averaged grid size in the level of $1 \mathrm{~cm} \times 1 \mathrm{~cm} \times 1 \mathrm{~cm}$ is set for the plasma region, which is more than enough to resolve the fast wave and to reach a good simulation convergence.

The rest of the paper is organized as follows. In section 2, a 2D COMSOL model solving the wave equation is developed and benchmarked against the Mie scattering theoretical model. The influence of filament size, filament density, number of filaments and distance between filaments on the scattering of fast wave are studied. In section 3, the influence of ELMs on the antenna power and antenna loading resistance are discussed with the experimental results in ASDEX Upgrade (AUG). In section 4, the influence of ELMs on the scattering of ICRF 
waves in realistic AUG geometries is studied by coupling the JOREK $[10,11]$ and RAPLICASOL $[12,13]$ codes. The nonlinear MHD code JOREK is used to calculate the 3D density during an ELM, and the 3D antenna code RAPLICASOL is used to calculate the wave fields and RF power flow. Finally, conclusions are given in section 5.

\section{Influence of filaments on wave fields}

In this section, a 2D COMSOL model solving the wave equation is developed and benchmarked against the Mie scattering theoretical model. Then, the influence of filament density, filament radius, number of filaments as well as the distance between filaments on RF wave scattering is studied with the Perfect Matching Layers (PML) boundary condition. Finally, simulations are done with the more realistic poloidal periodic boundary condition and filament density profile.

\subsection{Simulation model}

A 2D COMSOL model is built to simulate the interaction between RF waves and filaments in magnetized plasmas (Fig. 1(a)). In the model, the wave equation of the following form is solved:

$$
\nabla \times(\nabla \times \vec{E})=\frac{w^{2}}{c^{2}} \epsilon \vec{E}+i \omega \mu_{0} J_{e x t}
$$

in which $\epsilon$ is the cold plasma dielectric tensor and $J_{\text {ext }}$ denotes the antenna current. Here, the $\mathrm{x}, \mathrm{y}$ and $\mathrm{z}$ axes are representative of the radial, poloidal and toroidal directions in a tokamak, respectively. The filaments are assumed to have circular cross-sections in the $x-y$ plane, align with the magnetic field lines and be infinitely long in the z-axis direction. Thus, if we assume toroidal symmetry, the z-axis can be ignored and we only need to consider the physics in the $x-y$ plane. The left boundary of the simulation region is set with a line current density while the other three boundaries are set with PMLs. All waves being excited will eventually be absorbed by the PMLs. Since the radial propagation speed $\left(w / k_{r}\right)$ of the fast wave $\left(\sim 10^{6} \mathrm{~m} / \mathrm{s}\right)$ is much larger than the propagation speed of the filament $\left(\sim 10^{3} \mathrm{~m} / \mathrm{s}\right)$, the filament is considered to be stationary in the simulations. Cold plasma conditions are assumed and no RF wave power absorption is considered in the simulations. In section 2.2, constant densities are assumed both for the filament and background plasma. In section 2.3, a Gaussian density is defined for the filament. The grid (with triangular shape) resolution of the filament and background plasma is $3 \mathrm{~mm}$ and $1 \mathrm{~cm}$, respectively.

Previously, Ram et al. developed an analytical model [5] in which the solution to the wave equation for a plane wave which scatters at a filament is written as an infinite sum over azimuthal modes in cylindrical coordinates, akin to the classical "Mie" solution [14] for plane wave scattering at perfectly conducting spheres or cylinders. In our calculations, we used 41 azimuthal modes. The developed 2D COMSOL model is benchmarked with this analytical model by using the same plasma and wave parameters in both models: filament density $n_{e, F}=1 \times$ $10^{20} \mathrm{~m}^{-3}$, filament radius $r_{b}=0.02 \mathrm{~m}$, filament location $(\mathrm{x}, \mathrm{y})=(0,0 \mathrm{~m})$, background plasma density $n_{e, 0}=5 \times$ $10^{19} \mathrm{~m}^{-3}$, background magnetic field $B_{0}=-2.0 \mathrm{~T}$, ICRF wave frequency $\mathrm{f}=36.5 \mathrm{MHz}$, parallel wave vector $k_{z}=9$ $\mathrm{rad} / \mathrm{m}$ and current density $J_{s 0}=1 \mathrm{~A} / \mathrm{m}$. These parameters are similar to those of a typical $\mathrm{H}$-mode in AUG. The 
calculated results with the 2D COMSOL model and the analytical model show very good agreement (Fig. 1(b)), indicating that the 2D COMSOL model can be used with confidence.

(a) comsol model

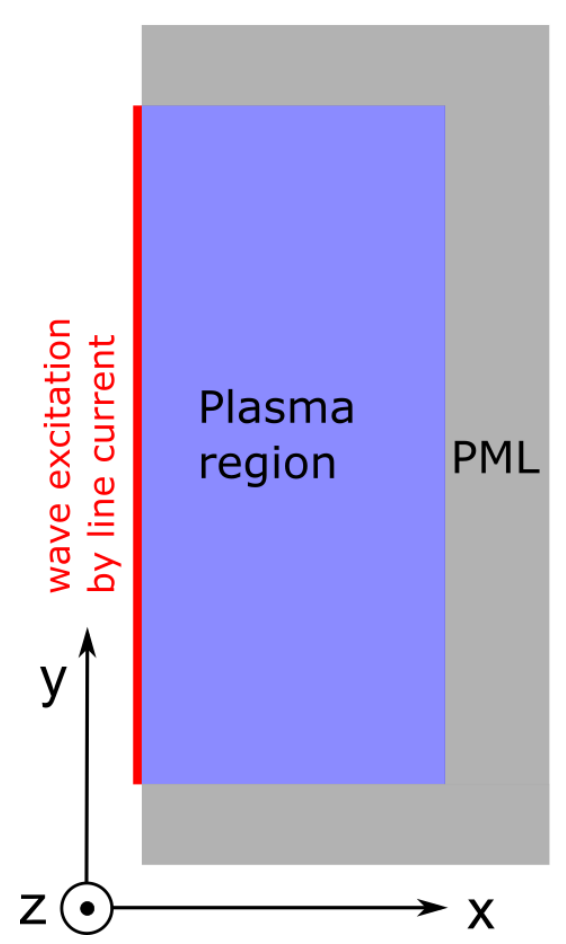

(b) benchmark with analytical model
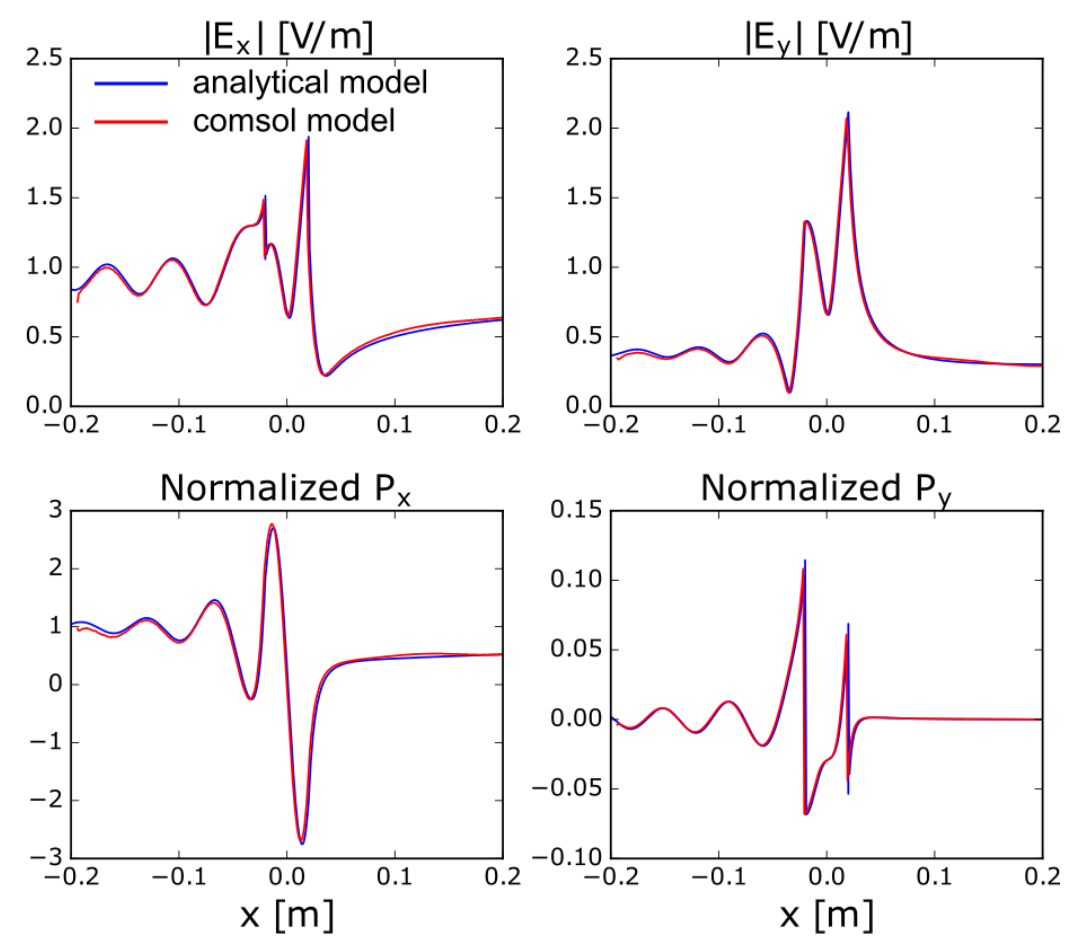

Figure 1. (a) The setup of the 2D COMSOL model. (b) Benchmark of the 2D COMSOL model with the analytical model for wave fields and Poynting flux in the midplane (i.e. $y=0 \mathrm{~m}$ )

\subsection{Simulations with PML boundary}

We start the simulations by considering a single filament and poloidal PML boundary condition. The current density is now set as $1 \mathrm{kA} / \mathrm{m}$, in same level as the AUG antenna straps. Other plasma and wave parameters are set as the same as section 2.1. In the following analysis, $\delta n_{e}=\left(n_{e, F}-n_{e, 0}\right) / n_{e, 0}$ and $d P_{x}=\left(P_{x, F}-P_{x, 0}\right) / P_{x, 0}$ are used to represent the perturbation of density and Poynting flux, respectively. Here, $n_{e, F}$ and $n_{e, 0}$ are the filament density and background density, $P_{x, F}$ and $P_{x, 0}$ are the radial Ponyting flux for the cases with and without filament, respectively.

To study the influence of filament density on RF wave scattering, the filament radius $(r=0.03 \mathrm{~m})$ is fixed and the filament density is considered as the only variable in the simulations. The results (Fig. 2(a)) clearly show that the RF wave scattering effect becomes stronger as the density perturbation in the filament becomes larger. A scattering cone with stripe structures of enhanced or reduced Poynting flux is developed around the filament. These stripe structures are correlated with the wavelength of the injected wave. As the filament density increases, the magnitude of perturbation increases while the shape of the scattering cone remains roughly the 
same. The largest perturbation of electric field and Poynting flux is located inside or in the periphery of the filament.

To study the influence of filament radius on RF wave scattering, the filament density $\left(n_{e, F}=1 \times 10^{20} \mathrm{~m}^{-3}\right)$ is fixed while the filament radius is considered as the only variable. The results (Fig. 2(b)) indicate that the wave scattering effect becomes larger as the filament radius increases. This scattering effect is obvious even if the filament radius (e.g. $r=0.01 \mathrm{~m}$ ) is an order of magnitude smaller than the wavelength of fast wave $\left(\lambda_{x} \sim 0.13 \mathrm{~m}\right)$. Again, a scattering cone with many stripe structures of enhanced and reduced wave fields is developed around the filament. The magnitude of perturbation increases as the filament radius increases while the shape of the scattering cone keeps roughly the same. The largest perturbation of electric fields and Poynting flux are located inside or in the periphery of the filament. Moreover, the shadowing effect in the backside of the filament with reduced magnitude of wave fields becomes stronger as the filament radius increases.

(a) Influence of filament density
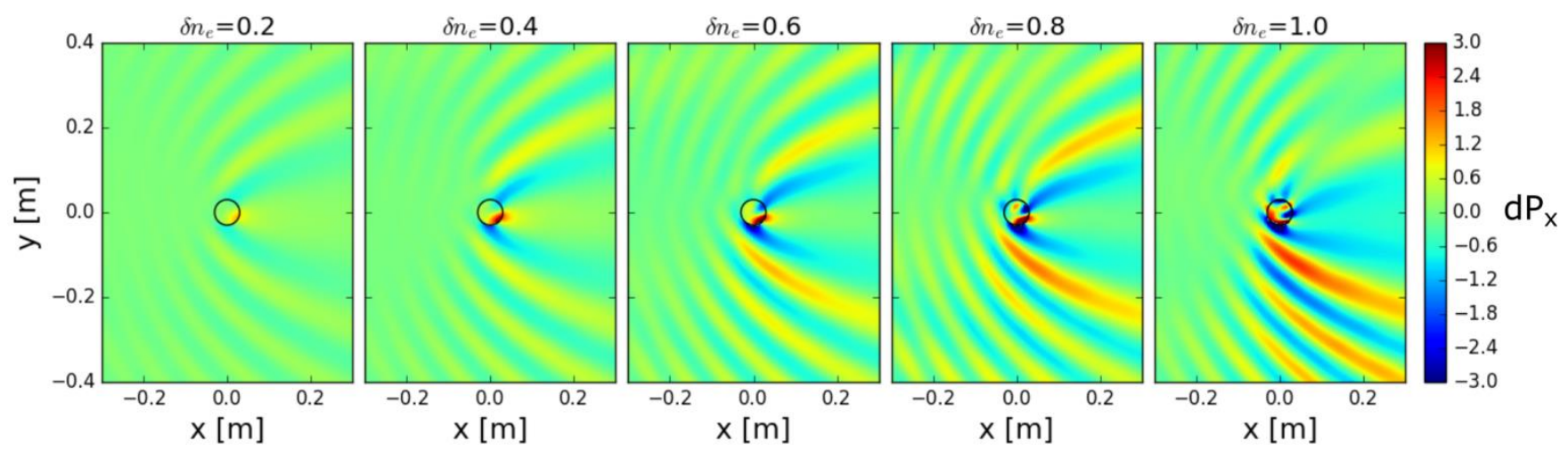

(b) Influence of filament radius
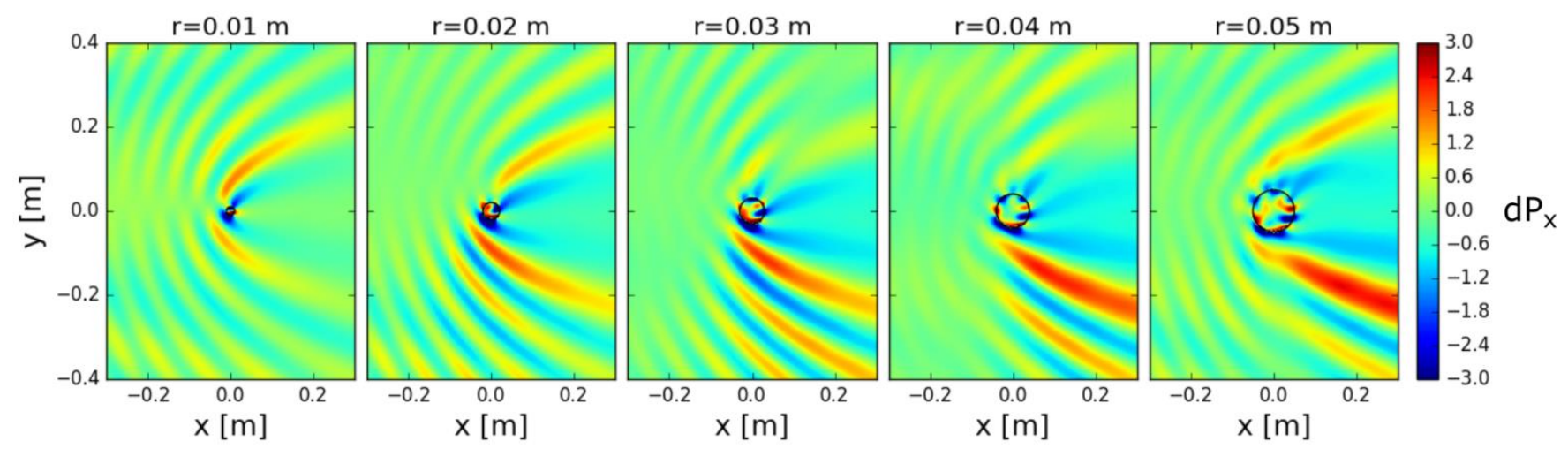

Figure 2. Influence of (a) filament density and (b) filament radius on the perturbation of Poynting flux $P_{x}$. The black circles depict the location of filaments. $\delta n_{e}$ is the density perturbation inside the filament and $r$ is the filament radius. 

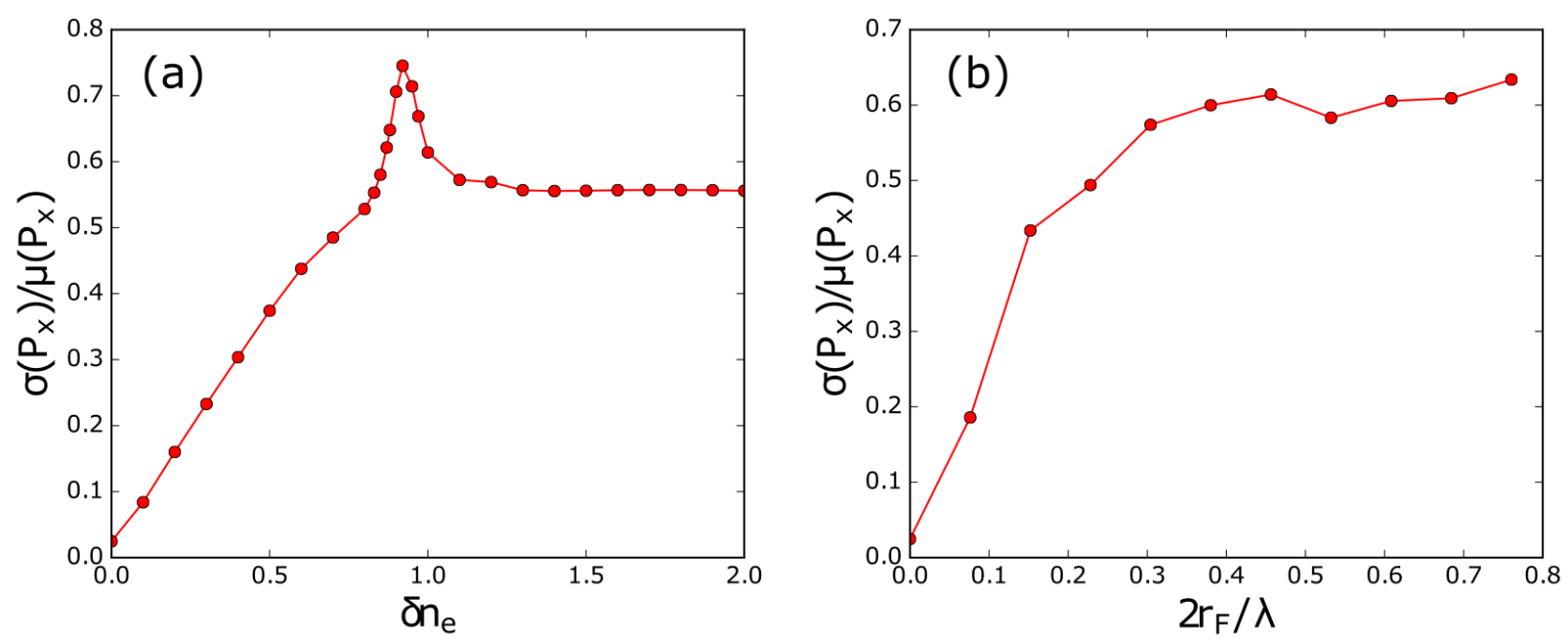

Figure 3. The normalized standard deviation of $P_{x}$ (normalized to the mean value of $P_{x}$ ) as a function of (a) density perturbation and (b) normalized filament diameter (normalized to the incident wavelength). The simulation region of $x=[-0.3,0.3 \mathrm{~m}]$ and $\mathrm{y}=[-0.5,0.5 \mathrm{~m}]$ is considered in the calculation. The filament radius $(\mathrm{r}=0.03 \mathrm{~m})$ is fixed in (a) and the filament density $\left(n_{e, F}=1 \times 10^{20} \mathrm{~m}^{-3}\right)$ is fixed in (b).

To have a more quantitative understanding, parameter scans of the filament density and filament radius $\left(r_{F}\right)$ are performed. Here, we use the normalized standard deviation of the radial Poynting flux $\sigma\left(P_{x}\right) / \mu\left(P_{x}\right)$ to characterize the level of wave scattering, in which $\mu\left(P_{x}\right)$ is the mean value of Poynting flux. The results (Fig. 3) indicate that $\sigma\left(P_{x}\right) / \mu\left(P_{x}\right)$ depends almost linearly on $\delta n_{e}$ before it gets saturated at $\delta n_{e} \geq 1$. Its dependence on the filament radius is more nonlinear for $2 r_{F} / \lambda$ is in the range of [0,0.4], then it also gets saturated when $2 r_{F} / \lambda$ further increases. In the Mie scattering theory, it is also shown that the Mie scattering efficiency will reach a saturation value if the sphere/filament size exceeds a threshold value [15].

The RF wave scattering by multiple filaments is much more complicated than by a single filament. Here, the influence of the number of filaments and the distance between filaments are studied. In the simulations, all filaments are identical and have a radius of $0.02 \mathrm{~m}$ and a density perturbation of 1 . When the distance between filaments is fixed $\left(d_{F}=0.1 \mathrm{~m}\right)$, the number of filaments is considered as the only variable. When the number of filaments is fixed $(\mathrm{N}=3)$, the distance between filaments is considered as the only variable. The simulation results (Fig. 4(a)) indicate that more filaments will lead to a stronger wave scattering effect. As the number of filaments increases, the back-scattering effect becomes stronger and the scattering cone which is usually seen in the single filament case becomes less prominent. The largest perturbation of the wave fields and Poynting flux is still close to the filaments. For a fixed number of filaments (Fig. 4(b)), an increase of the distance between filaments will lead to an increase of the global perturbation of wave fields. More specifically, when the distance between the filaments is small, for instance when $d_{F} \leq 0.1 \mathrm{~m}$, the resulting effect is more like that caused by a single large filament. A scattering cone can still be clearly identified, with the largest perturbation of wave fields locates close to the filament. When this distance is larger, for instance when $d_{F} \geq 0.2 \mathrm{~m}$, the scattering cone becomes less obvious and the wave fields are more stochastic. The largest perturbation of wave fields does not necessary locates near the filaments any more. 
(a) Influence of number of filaments
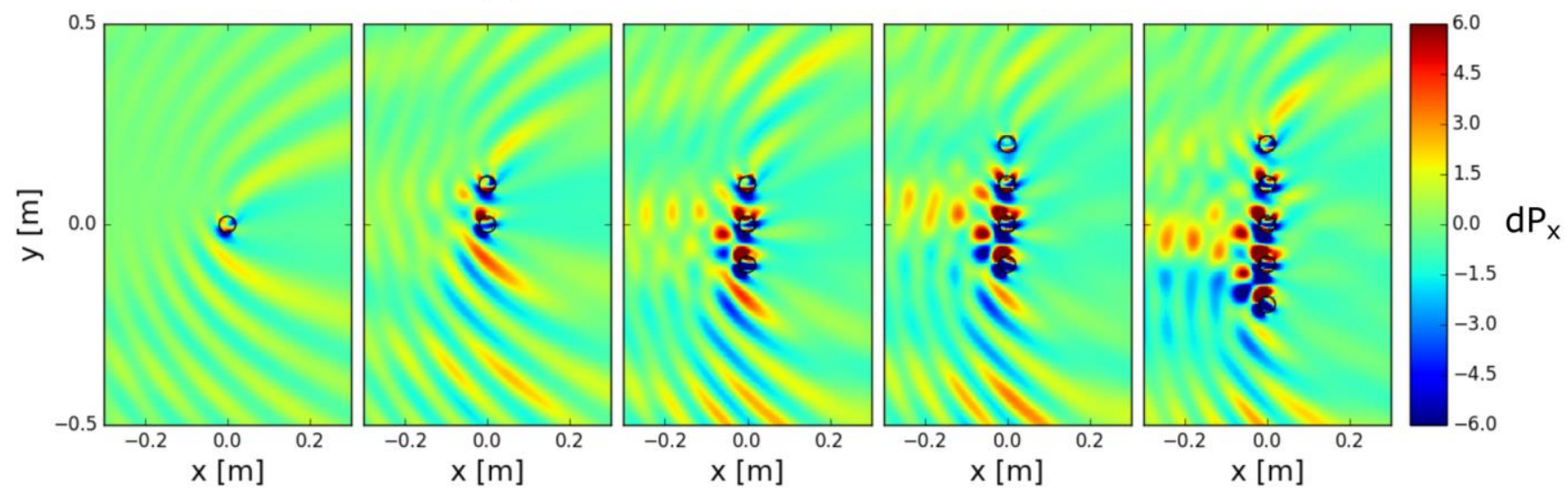

(b) Influence of distance between filaments
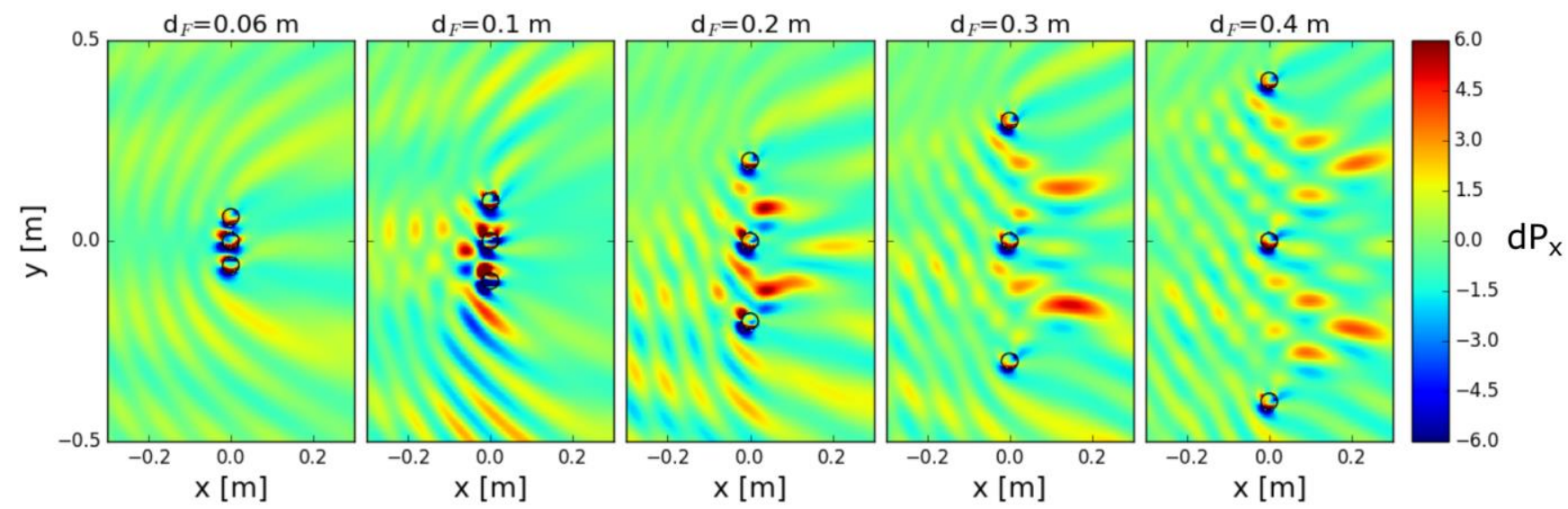

Figure 4. Influence of (a) number of filaments and (b) distance between filaments on the relative change of the Poynting flux $P_{x}$. The black circles depict the location of filaments. A constant distance of $d_{F}=0.1 \mathrm{~m}$ between the filaments is used in Fig. 4(a).

To have a more quantitative understanding of the influence of the number of filaments and the distance between filaments, the normalized standard deviation of the radial Poynting flux (i.e. $\sigma\left(P_{x}\right) / \mu\left(P_{x}\right)$ ) is calculated. The results (Fig. 5) show that $\sigma\left(P_{x}\right) / \mu\left(P_{x}\right)$ depends roughly linearly on the number of filaments. Its dependence on the distance between filaments is more complicated. It firstly increases linearly and peaks at $d_{F} / \lambda \approx 0.4$. At this point, the separated filaments still act like a single filament. Once $d_{F} / \lambda>0.4$, part of the wave can penetrate the gaps between the filaments and the major perturbation starts to shift radially away from the filaments. As a result, $\sigma\left(P_{x}\right) / \mu\left(P_{x}\right)$ starts to vary around 1.

In reality, the density perturbation, the filament radius and the distribution of SOL filaments are much more complicated than what we assumed in this section. The wave scattering will be much more sophisticated. Thus more realistic simulations are required. The corresponding simulation strategy and results will be shown in section 4. 

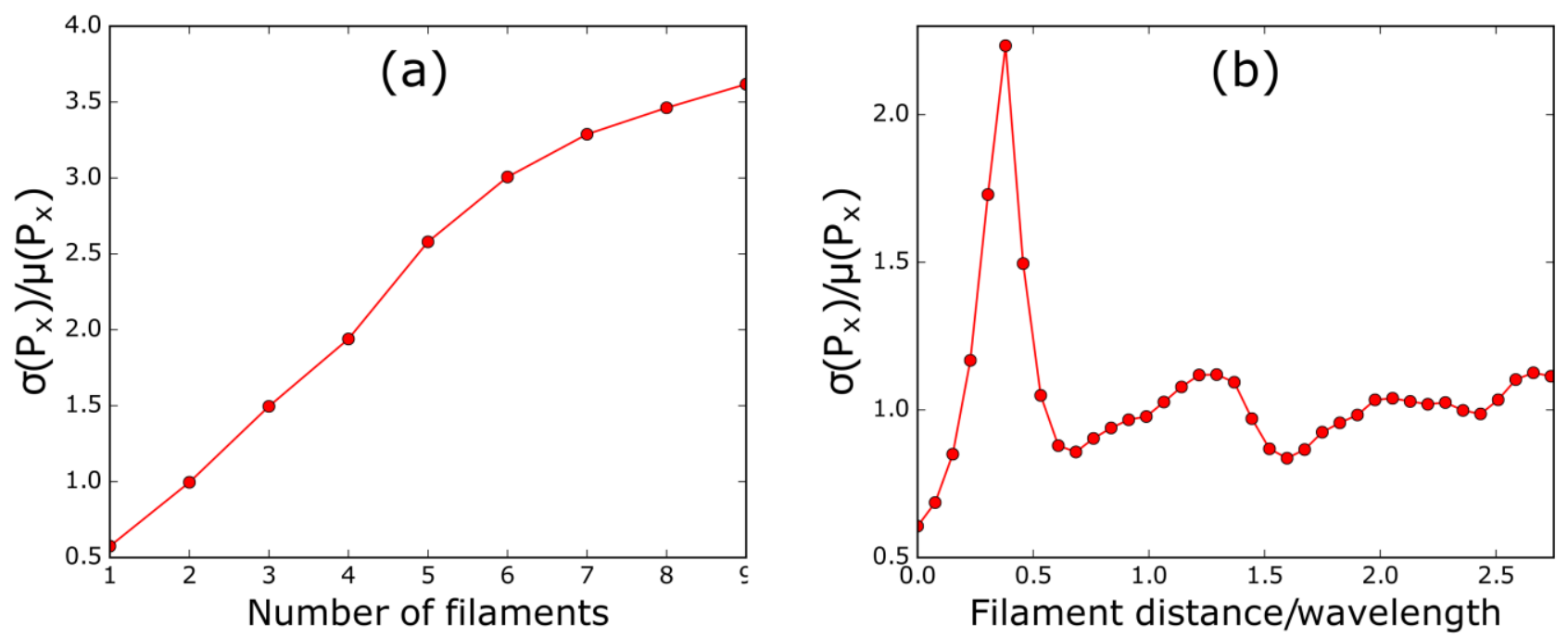

Figure 5. The normalized standard deviation of $P_{x}$ (normalized to the mean value of $P_{x}$ ) as a function of (a) the number of filaments; (b) the normalized distance between filaments (normalized to the incident wavelength). A radial and poloidal extent of $x=[-0.3,0.3 \mathrm{~m}]$ and $y=[-0.5,0.5 \mathrm{~m}]$ are considered in the calculation. All filaments are identical and have a radius of $0.02 \mathrm{~m}$ and a density perturbation of 1 . The distance between filaments $\left(d_{F}=0.1 \mathrm{~m}\right)$ is fixed in (a) and the number of filaments $(\mathrm{N}=3)$ is fixed in (b).

\subsection{Simulations with periodic boundary}

In this section, the poloidal PML boundary condition is changed to a poloidal periodic boundary condition (Fig. 6(a)). With this boundary condition, both the filament and wave are assumed to be poloidal periodic. In addition, a Gaussian density which is thought to be more realistic [16] is used to set the filament density. A constant background density as before is still used. The density transition at the filament - background plasma boundary becomes smooth now. The applied Gaussian density has the following form:

$$
n_{e, G}=n_{e_{-} \max } * \exp \left(-\frac{\left(x-x_{0}\right)^{2}}{2 \sigma^{2}}-\frac{\left(y-y_{0}\right)^{2}}{2 \sigma^{2}}\right)
$$

in which $\left(x_{0}, y_{0}\right)$ is the center of filament, $\sigma$ is the standard deviation of the Gaussian density, $n_{e_{-}} \max$ is the maximum density and locates at the center point. Here, we use the maximum density perturbation $\delta n_{e_{-} \max }$ and $\sigma$ to control the filament density and filament radius, in which $\delta n_{e_{-} \max }=\left(n_{e_{-} \max }-n_{e, 0}\right) / n_{e, 0}$. Other plasma and wave parameters are set as the same as those in section 2.1 . 


\section{(a) comsol model}

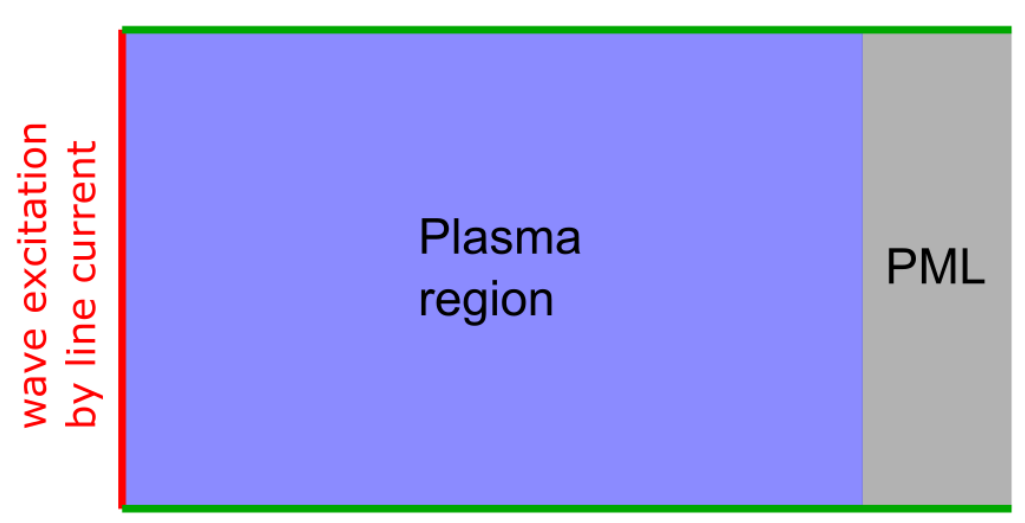

(b) Gaussian density
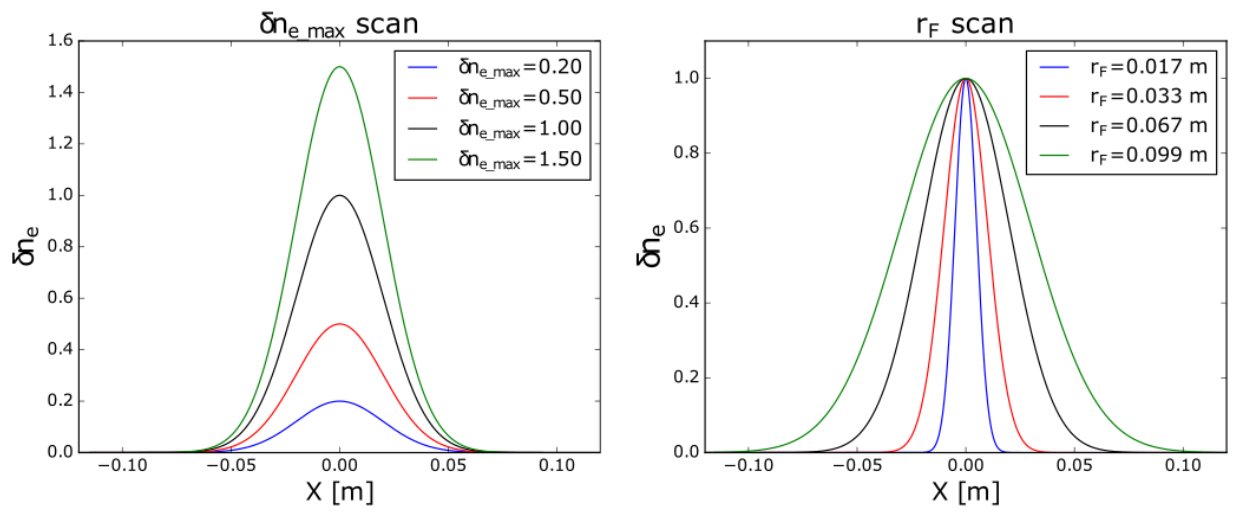

Figure 6. (a) The 2D COMSOL model with poloidal periodic boundary condition. The other settings in the COMSOL model are the same. (b) The Gaussian density used in the filament. $\delta n_{e}$ is the density perturbation relative to the background plasma.

Similar to the previous section, the influence of filament density and filament radius on RF wave scattering is studied. In the former study, the filament radius is kept constant $\left(r_{F}=0.067 \mathrm{~m}\right.$, i.e. $\left.\sigma=0.02\right)$ while the filament density is the only variable. In the later study, the filament density $\left(\delta n_{e_{-} \max }=1.0\right)$ is kept constant while the filament radius is the only variable. The results (Fig. 7) show that the effect of wave scattering becomes stronger as the filament density or the filament radius increases. The perturbation of Poynting flux originates near the filament can radially extend by $\sim 0.5 \mathrm{~m}$. For a tokamak with a minor radius of $0.5 \mathrm{~m}$, the RF wave scattering caused by filament in the SOL can indeed penetrate into the core plasma. This means the perturbed wave fields can reach the resonance layer and influence the core heating. Moreover, the largest perturbation of the Poynting flux does not necessary locates in the vicinity of the filament. Scattering cones can still be identified, and their shapes depend strongly on the filament radius.

To have a more quantitative understanding, parameter scans of the filament density and filament radius are performed. Again, the normalized standard deviation of Poynting flux (i.e. $\sigma\left(P_{x}\right) / \mu\left(P_{x}\right)$ ) is calculated. The results (Fig. 8) show that as the filament density and filament radius increases, $\sigma\left(P_{x}\right) / \mu\left(P_{x}\right)$ increases nonlinearly. This increase is prominent when $\delta n_{e_{-} \max }$ is in the range of $[0,0.9]$ or when $2 r_{F} / \lambda$ is in the range of $[0,0.8]$, and 
becomes smaller as $\delta n_{e_{-} \max }$ or $2 r_{F} / \lambda$ further increases. The parameter scan of the distance between filaments is similar to the case with constant filament density, i.e. Fig. $5 \mathrm{~b}$.

(a) Influence of filament density
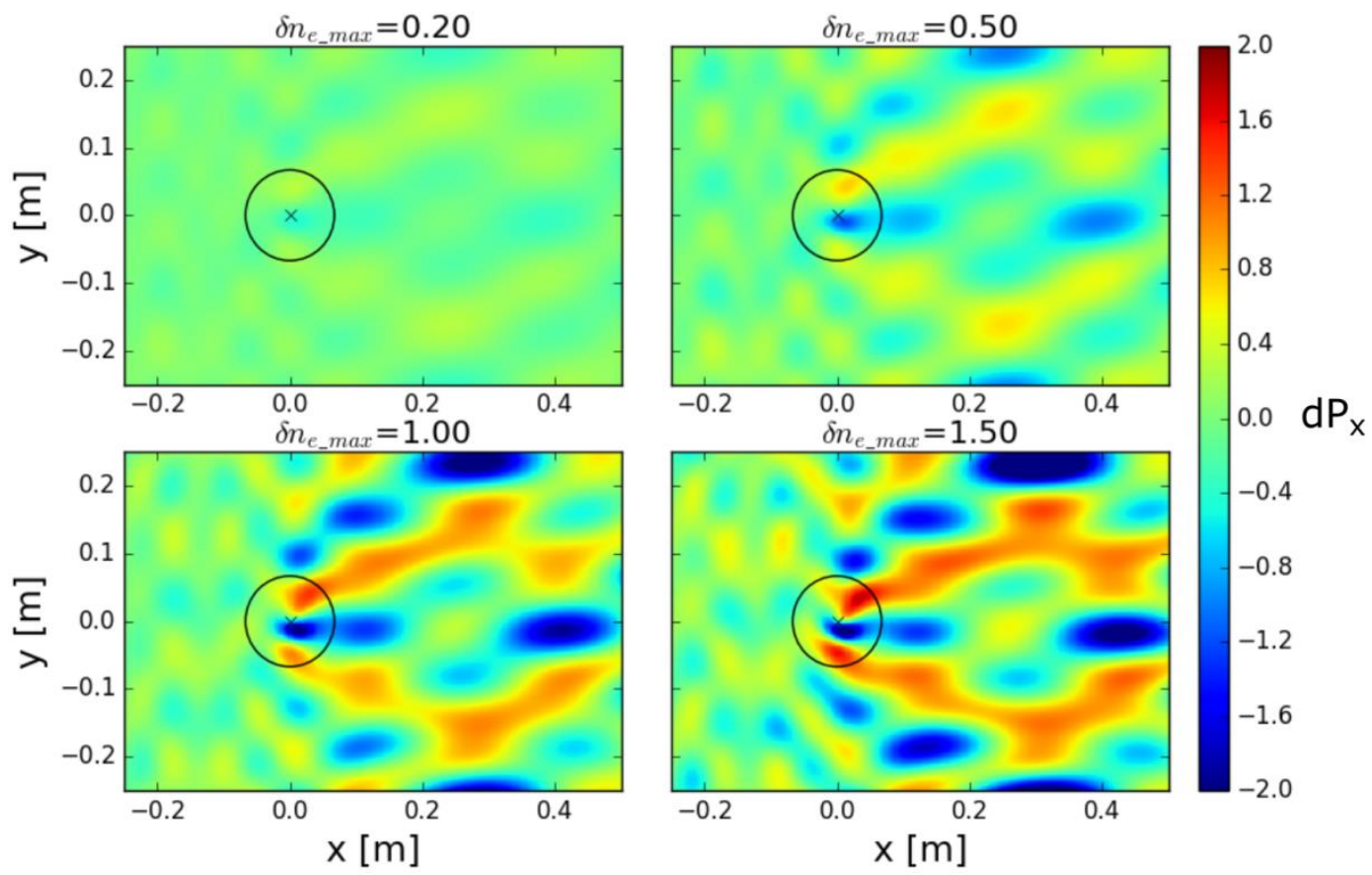

(b) Influence of filament radius
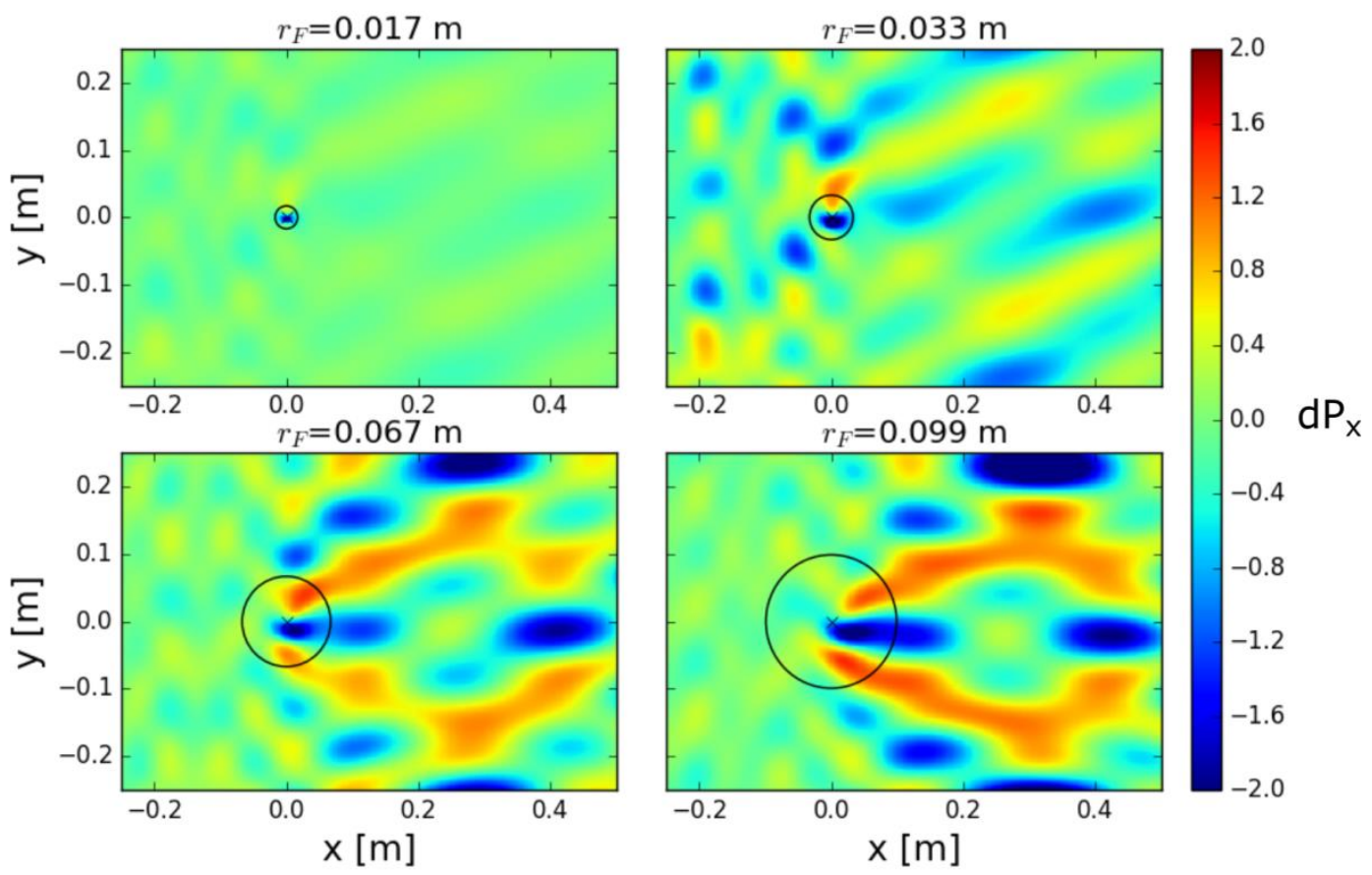

Figure 7. Influence of (a) filament density and (b) filament radius on the relative change of the Poynting flux $P_{x}$. The corresponding filament densities are shown in Fig. 6 (b). 

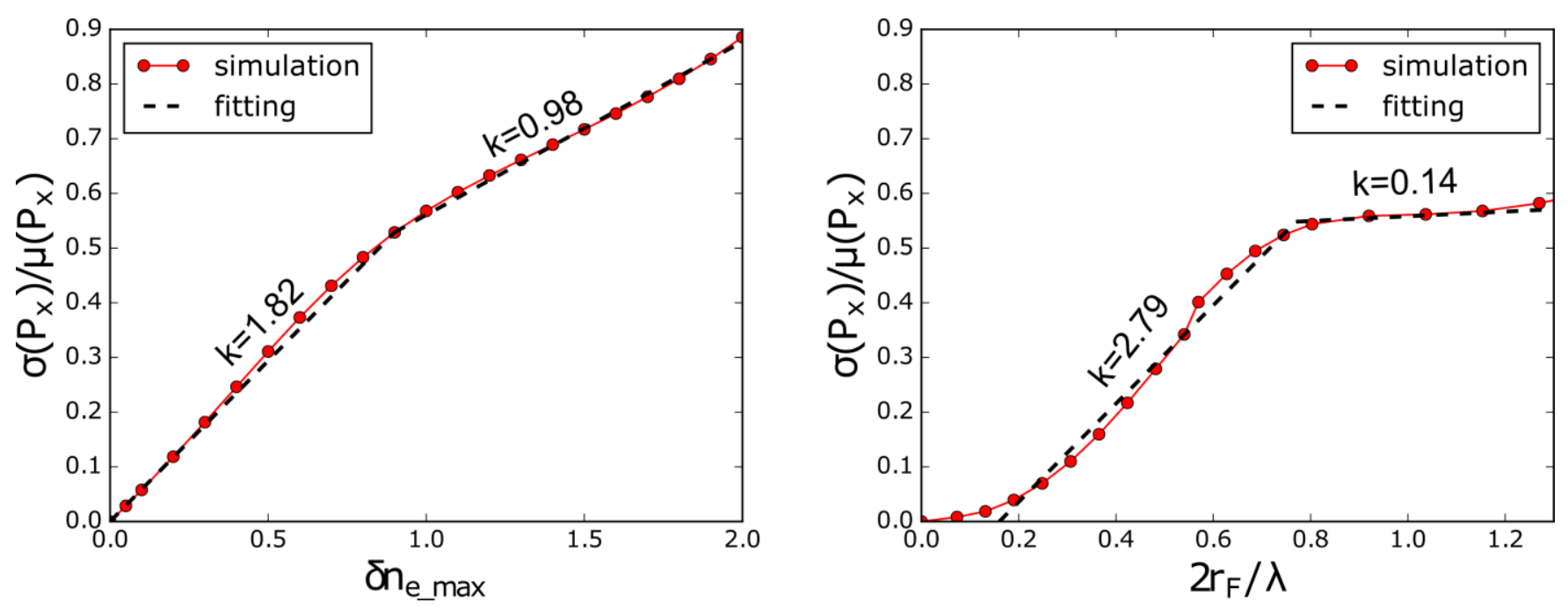

Figure 8. The normalized standard deviation of $P_{x}$ (normalized to the mean of $P_{x}$ ) as a function of the density perturbation inside the filament and the ratio between the filament diameter and wavelength. The $k$ values shown in the figures are the slopes of the fitted lines. The filament radius $\left(r_{F}=0.067 \mathrm{~m}\right)$ is fixed in the left subfigure and the maximum filament density $\left(\delta n_{e_{-} \max }=1.0\right)$ is fixed in the right subfigure.

\section{Experimental results}

The RF power produced by the RF generators $\left(P_{\text {generator }}\right)$ is delivered to the antenna through the transmission line. A matching network located close to the antenna is used to match the impedance between the antenna and the transmission line. A part of the RF power is reflected ( $\left.P_{\text {reflected }}\right)$ on the generator side of the matching network because of the imperfect impedance matching, especially when the matching condition is dramatically changed by the SOL plasma during ELMs. The rest of power, i.e. the difference between the forward power and the reflected power on the matched side of the network, is the power delivered to the antenna $\left(P_{\text {antenna }}\right)$. Most of the RF power is coupled to the plasma ( $P_{\text {coupled }}$ ) while a small part is lost in the antenna and in the RF transmission lines on the RF conductors and due to the RF losses in the ceramics ( $\mathrm{P}_{\text {loss_RF_lines }}$ ). Experimentally, the effective coupling resistance is estimated by $R_{c}=2 \cdot P_{\text {coupled }} \cdot Z_{0}^{2} / V_{\text {max }}^{2}$, where $V_{\text {max }}$ is the maximum $\mathrm{RF}$ voltage in the unmatched transmission line. The power lost in the unmatched line is estimated by $P_{\text {loss_RF_lines }}=R_{\text {vac }} \cdot V_{\text {max }}^{2} /\left(2 \cdot Z_{0}^{2}\right)$, where $R_{v a c}$ is measured in vacuum case without any plasma. A schematic of the RF power flow is shown in Fig. 9(a). 
(a)

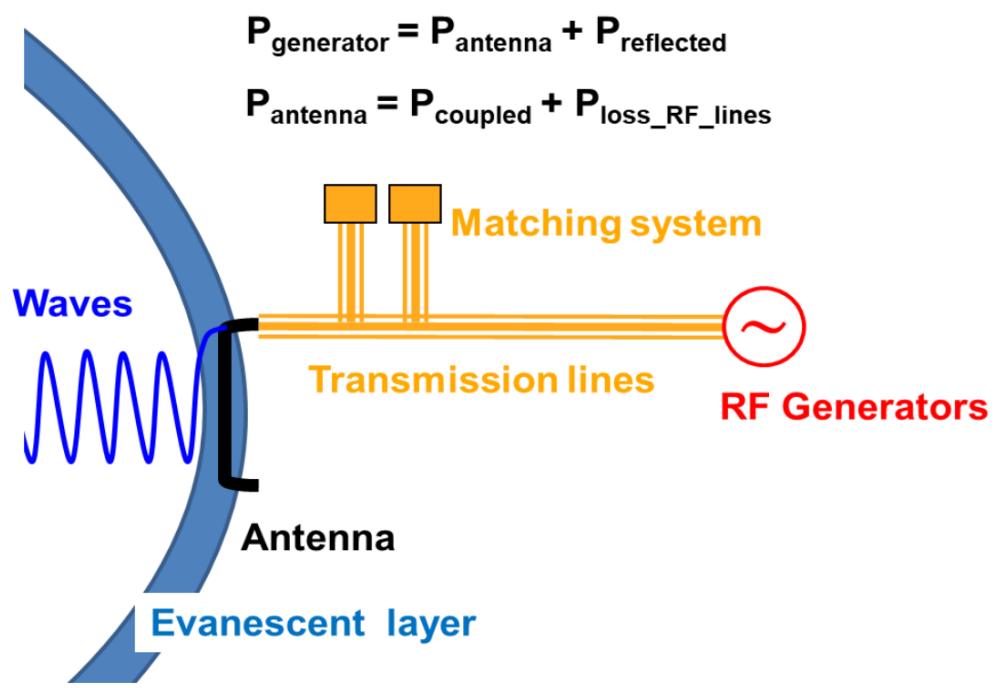

(b) $\# 35173$

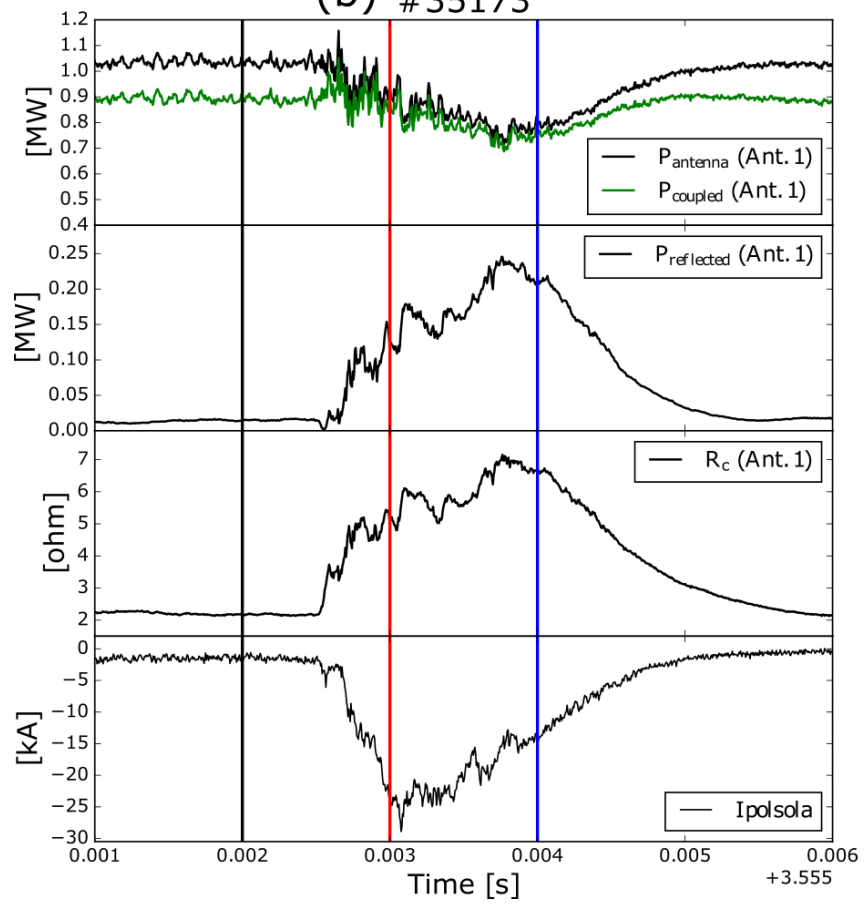

(c)

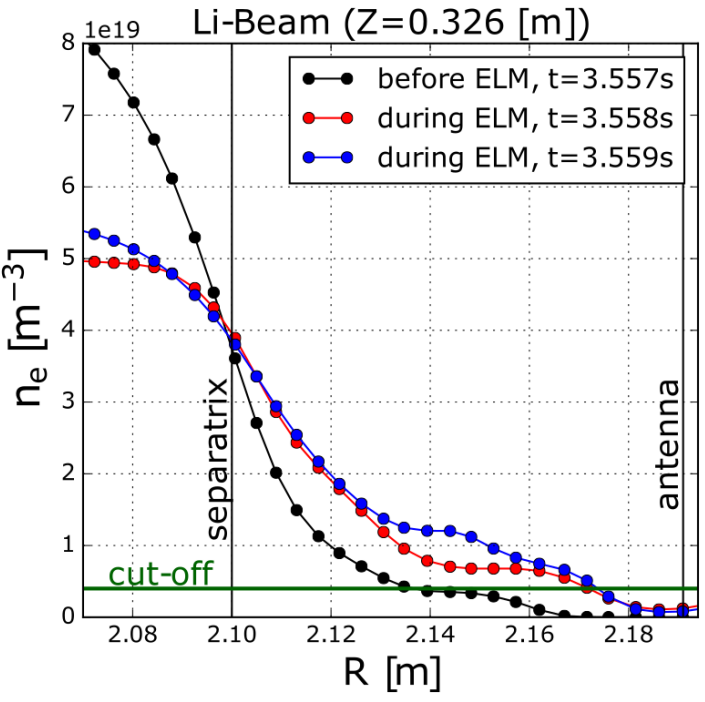

Figure 9. (a) RF power flow from the RF generators to the plasma. (b) The power reaching the antenna, the coupled power, the reflected power in the transmission line, the antenna loading resistance of antenna 1 and the outer divertor shunt current (current between a divertor plate and the ground) during an ELM. (c) The corresponding density profiles measured by Lithium beam diagnostic.

Filaments exist in all plasma scenarios while ELMs are usually found in $\mathrm{H}$-mode plasmas. ELMs are believed to be caused by peeling-ballooning instabilities $[17,18]$. They can lead to a collapse of the pedestal and a flattening of edge density profile. A large amount of plasma is pumped out into the SOL within milliseconds, in the form of big filaments. The SOL density during an ELM is different from that during the calmer inter-ELM regime, which 
has consequences w.r.t. ICRF wave behavior during ELMS, and changes the amount of ICRF power that reaches the core plasma. This is because the time-evolving density profile leads to a different impedance matching between the transmission line and antenna. The matching is usually chosen to fit better the periods between ELMs, in order to deliver the most energy over the ICRF pulse to the plasma. As a result, less power usually reaches the antenna during ELMs. In addition, the density modification can also change the amount of power coupled from the antenna to the plasma. This is due to the fact that the fast wave is only propagating when the plasma density is larger or equal to its cut-off density (usually in the level of $1 \times 10^{18} \mathrm{~m}^{-3}$ ). However, there is often an evanescent layer in the plasma periphery where the plasma density is lower than the cut-off density. The fast wave decays exponentially in this layer and has to tunnel through it. To a first approximation, the

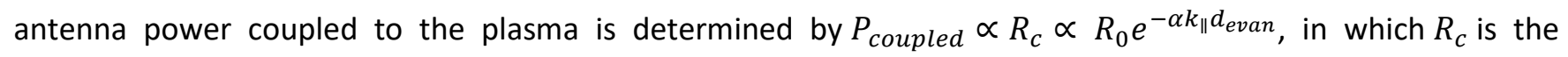
antenna loading/coupling resistance, $\alpha$ is the tunneling factor, $k_{\|}$is the parallel wave number and $d_{\text {evan }}$ is the width of the evanescent layer. $R_{0}$ is the reference coupling resistance when $d_{\text {evan }}=0$. During an ELM, the SOL density is on average increased. Consequently, the evanescent layer of the fast wave becomes narrower and more power can be coupled from the antenna to the plasma. The final coupled power to the plasma during ELMs is thus a combination of decreased antenna power due to the mismatch and an increased antenna coupling due to increased SOL density.

An example of the influence of an ELM on the antenna power and the effective coupling resistance in AUG is shown in Fig. 9(b). It is indicated that the power reaching the antenna is reduced, and the amount of reduced power $(\sim 0.25 \mathrm{MW})$ is about equal to the reflected antenna in the transmission line. On the contrary, the coupling resistance increases dramatically from $\sim 2 \Omega$ before the ELM to $\sim 5 \Omega$ during the ELM. This is consistent with the change of the density profiles and the width of evanescent layer, as shown in Fig. 9 (c). Compared to the density profile before the ELM, the density profile during the ELM shows a significant decrease of pedestal density and a strong increase of SOL density. Consequently, the width of evanescent layer decreases from $\sim 0.054 \mathrm{~m}$ to $\sim 0.017 \mathrm{~m}$. If using a simple experimental formula $P_{\text {coupled }} \propto R_{0} e^{-18 d_{\text {evan }} \text {, then the calculated }}$ coupling resistance based on the density profile is increased by $100 \%$. This is lower than the measured value of $150 \%$. It is suspected that the deviation of the measured and calculated coupling resistance is due to the following reasons: 1 . The density gradient also plays an important role in determining the coupling resistance, which is neglected in the calculations; 2. A 1D density profile is used in the calculation, while the realistic 3D density during an ELM is highly inhomogeneous spatially. The final coupled power from the antenna to the plasma (i.e. the green line in Fig. 9(b)), if counting both the decreased power reaching the antenna and the increased coupling resistance, decreases by $0.15 \mathrm{MW}$ (namely by $16.7 \%$ ) during the ELM.

Besides the influence discussed above, ELMs can also induce prominent RF wave scattering. ELM filaments usually have much larger sizes and density perturbations than filaments resulting from drift-wave turbulence. It is expected that the wave scattering effect during ELM is much larger than that in-between ELMs. However, it is difficult to measure the ELM filaments and the scattering of RF waves with the current diagnostics. Nevertheless, we can still characterize and understand this with realistic and comprehensive simulations, as will be shown in the next section. 


\section{Realistic simulations on ASDEX Upgrade}

\subsection{Simulations setups}

ELMs can cause large density perturbations $\left(\delta n_{e}\right)$ in the SOL, usually in the form of density filaments. The induced ELM filaments typically have a spatial size of a few centimeters and a density perturbation in the level of $100 \%$. Besides, density holes with negative density perturbations can also be generated. Both density filaments and density holes can cause a global perturbation of wave fields. To have a detailed understanding of the influence of ELMs on RF wave scattering in realistic tokamak geometry, the 3D reduced MHD code JOREK is used to calculate the $3 \mathrm{D}$ SOL density distribution during ELMs, which is then used in the 3D ICRF antenna code RAPLICASOL to calculate the RF wave fields. The basic principle of the simulation flow is demonstrated in Fig. 10.

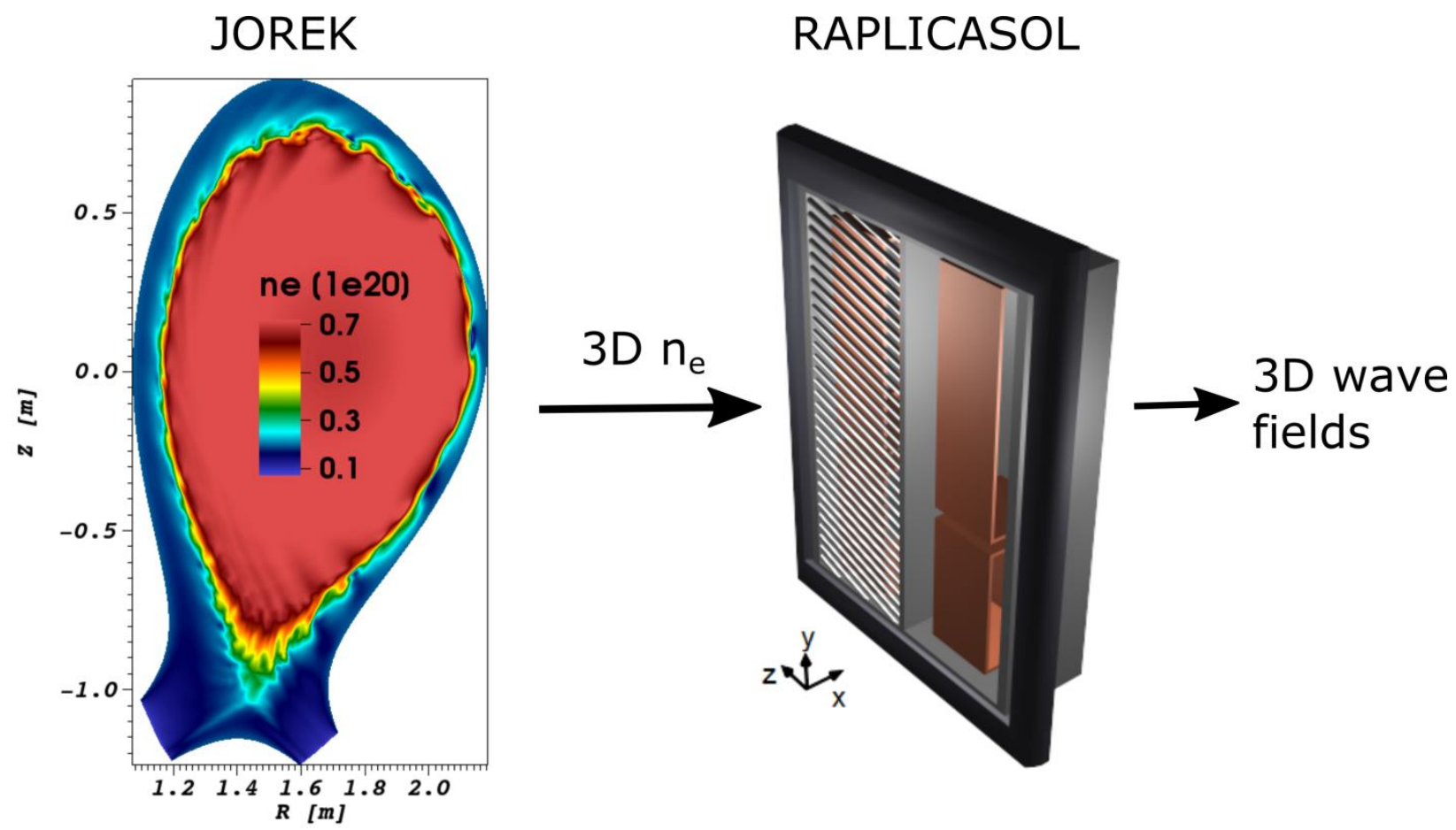

Figure 10. Simulations by coupling the MHD code JOREK and the ICRF antenna code RAPLICASOL. JOREK uses a toroidal coordinate system $(R, Z, \Phi)$ and RAPLICASOL uses Cartesian coordinates, in which the $(x, y, z)$ axis represent the (radial, poloidal, toroidal) directions.

The JOREK code $[10,11]$ is a thoroughly validated non-linear 3D visco-resistive MHD code. The reduced MHD [19] model with the extension for two-fluid diamagnetic effects [20] is used for the present work. Said model evolves the plasma density, poloidal magnetic flux, plasma temperature, electrostatic potential, and ion velocity parallel to the magnetic field $\mathbf{B}$ in time. With a set of given initial conditions (profiles of density, temperature, and poloidal current, toroidal magnetic field amplitude, and poloidal magnetic flux at the computational boundary), the evolution of the aforementioned variables is calculated. Additionally, profiles of perpendicular (to B) particle and heat diffusion coefficients are assigned to account for transport by neoclassical effects and small-scale turbulence, which is not captured by the MHD model. Finally, parallel heat diffusion according to the 
Spitzer-Härm model is included, leading to heat diffusion anisotropy in the order of $10^{8} \ldots 10^{10}$ across the plasma. The bootstrap current is evolved consistently to the density and temperature profiles. Sheath boundary conditions are applied at the divertor targets.

For the present study, two ELM crashes are simulated with JOREK for an AUG discharge (\#33616) with $B_{t}=-2.5 \mathrm{~T}, I_{p}=0.8 \mathrm{MA}$ and $q_{95}=5.2$. These correspond to a high SOL density case reproduced from Ref. [21] and to a low SOL density case simulated by reducing the fixed boundary condition for the density from Ref. [21]. The simulations encompass all the confined plasma, the scrape-off layer (SOL), and the private flux region below the $X$-point, and they contain all the even toroidal mode numbers up to $n=12$. The post-ELM pedestal recovery is simulated by heat and particle sources, which increase the pressure gradient at the edge of the confined region - which itself drives a large bootstrap current density and strong $E \times B$ and diamagnetic flows - until the peeling-ballooning stability threshold is crossed. Once the plasma crosses this threshold, small amplitude nonaxisymmetric perturbations begin to grow exponentially with a growth rate increasing over time due to the continuing pedestal build-up. When these plasma instabilities become large enough to affect the plasma background, the different toroidal mode numbers interact in a strongly non-linear way and cause significant losses of thermal energy and particle content ( $\sim \%$ for the high SOL density case and $\sim 15 \%$ for the low SOL density case) by convection and transport along the stochastic field lines. This constitutes the simulated ELM crashes, which have experimentally relevant durations of a few hundred microseconds.

RAPLICASOL is a finite-element solver based on COMSOL. It solves 3D full-wave Maxwell's equations in frequency-domain in the cold plasma approximation in the neighborhood of a realistic antenna. PML boundary condition is assumed at the core plasma boundary as well as the top and bottom simulation boundary. By putting a certain amount of power or voltage on the antenna ports, the straps excite waves which then propagate toward the plasma core. In our simulations, the flat AUG antenna model which is benchmarked with $3 D$ density is used. The newly built 3D curved antenna model still need more benchmarking before it can be used with confidence. Since the radial outmost density (in the far SOL and close to the leading edge of antenna limiters) from the JOREK simulations is $\sim 2 \times 10^{19} \mathrm{~m}^{-3}$ in the high boundary density case and $\sim 2.1 \times 10^{18} \mathrm{~m}^{-3}$ in the low boundary density case, the slow wave does not propagate at and beyond these densities and is thus not considered in our study. The region between the antenna strap and the outmost density position is filled with vacuum. In the RAPLICASOL simulations, only the 3D density profiles are taken from the JOREK simulations. The other important parameters set are: 1 kilovolt at each port of the 2-strap antenna; ICRF wave frequency $f_{I C R F}=36.5 \mathrm{MHz}$; central magnetic field $\mathrm{B}_{0}=-2.5 \mathrm{~T}$; a $5 \%$ Hydrogen minority in a bulk Deuterium plasma; radial, poloidal and toroidal extensions of the simulated region are $0.36 \mathrm{~m}, 1.86 \mathrm{~m}, 2.23 \mathrm{~m}$, respectively. A standard 2strap antenna is considered.

In the following subsections, two cases are studied: one with a fixed high boundary density (larger than the fast wave cut-off density) and smaller ELM crash; the other one with a fixed low boundary density (lower than the fast wave cut-off density) and larger ELM crash. In each case, the JOREK density profiles at a series of time slices (extracted during a time interval of $0.4 \mathrm{~ms}$ ) are used in the RAPLICASOL simulations, such that the evolution of the change of the wave fields during an ELM crash can be simulated. 
Since JOREK uses a toroidal coordinate system $(R, Z, \Phi)$ and RAPLICASOL uses Cartesian coordinates $(x, y, z)$ and a flat antenna model, coordinate transformation has to be performed. This coordinate transformation is done at every magnetic flux surface with the following formula:

$$
(\mathrm{x}, \mathrm{y}, \mathrm{z})=\left(R_{Z=Z_{0}}, \int_{Z_{0}}^{Z} s d s, R_{0} \Phi\right)
$$

in which $\left(R_{0}, Z_{0}\right)=(2.2,0.0 \mathrm{~m})$ is the center of the antenna limiter, $s$ is the poloidal length along the magnetic flux surface, and $R_{Z=Z_{0}}$ is the radial coordinate of the respective flux surface in the midplane $\left(Z=Z_{0}\right)$.

\subsection{Simulations with a high boundary density}

In this section, the case with a fixed high boundary density $\left(2 \times 10^{19} \mathrm{~m}^{-3}\right)$ and small ELM crashes is studied. The fixed high boundary density is larger than the fast wave cut-off density $\left(\sim 4 \times 10^{18} \mathrm{~m}^{-3}\right)$. As a result, the influence of the ELM onto the width of the evanescent layer cannot be taken into account. Nevertheless, the influence of the ELM onto RF wave propagation, which is the main topic of the paper, can still be well studied in this section. Fig. 11 shows the evolution of the calculated outer midplane density profile and the magnetic energy of the perturbations during an ELM. It can be seen that the MHD modes with toroidal mode number of $\mathrm{n}=2$ and 4 play a dominant role during the ELM crash, similar to experimental observations described in Ref. [22]. During the peak of ELM ( $\mathrm{t}=\mathrm{t} 7)$, the pedestal density is decreased dramatically while the SOL density is increased. The calculated radial wavelength of the fast wave with these density profiles is $\lambda_{\perp} \approx 0.13 \mathrm{~m}$, which is in the same level as the size of ELM filaments $(\sim 0.1 \mathrm{~m})$. Thus an obvious wave scattering by ELMs is expected, but the scattering structures may be quite different to what we see in section 3 since the distribution of the ELM filaments is much more complicated than before. 

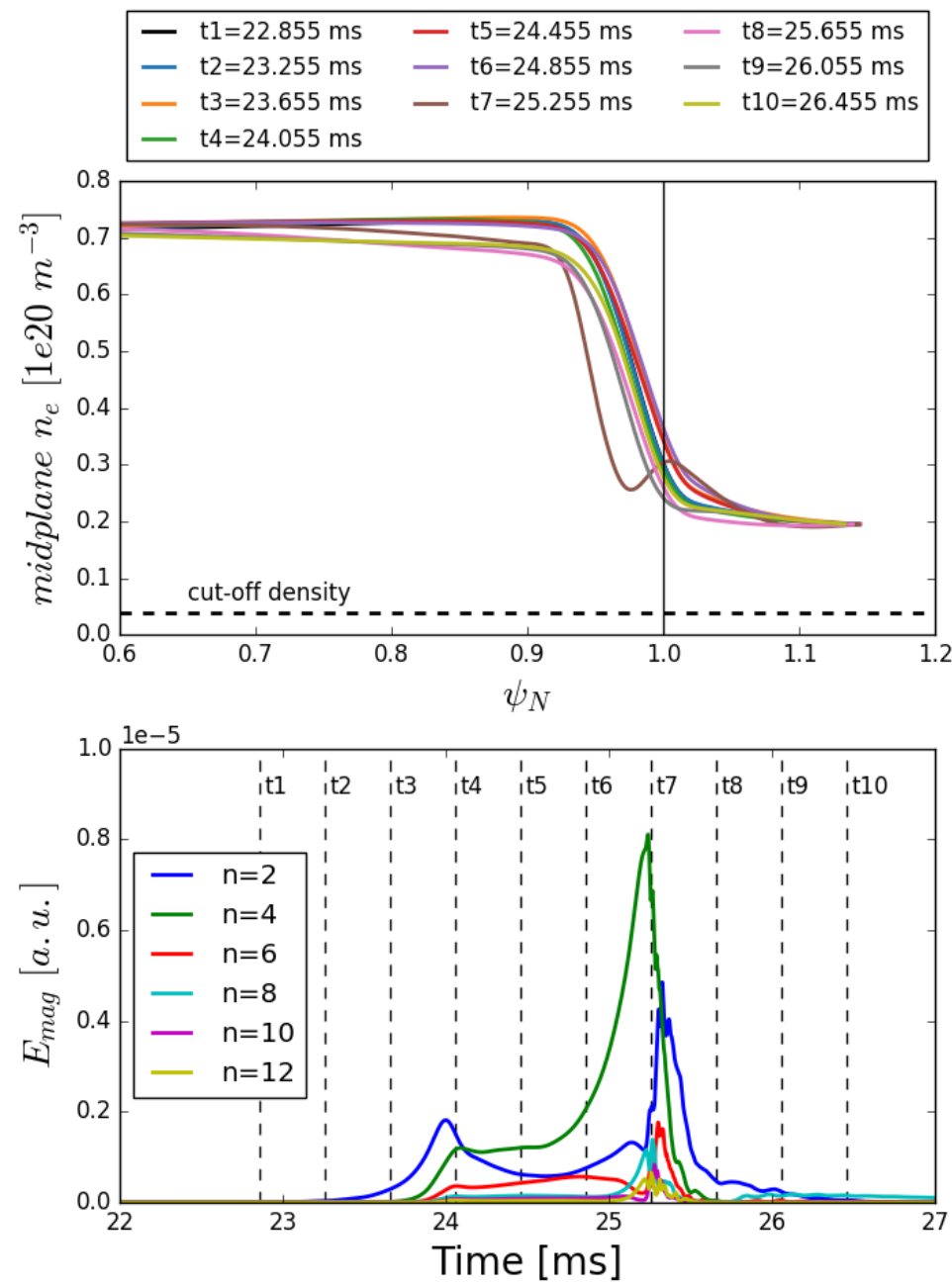

Figure 11. (a) The outer midplane density profiles with a high fixed boundary density. (b) The magnetic energy of the non-axisymmetric perturbations during an ELM.

The 3D wave fields at different time slices during an ELM crash are then calculated by RAPLICASOL. A comparison of the calculated wave fields at time slices before the ELM ( $\left.t=t_{1}\right)$ and during the ELM peak $\left(t=t_{7}\right)$ is shown in Fig. 12. The poloidal cross-sections are taken at the toroidal center of the right strap ( $z=0.2 \mathrm{~m})$. Since this strap has a longer current component in the upper side of the antenna than the lower side, the produced wave fields thus have a larger poloidal extension in the upper side of the antenna. At the time slice before the ELM burst $\left(t_{1}=22.855 \mathrm{~ms}\right)$, the density is poloidally rather homogeneous. The many small spike structures in the wave field just in front of the antenna $(2.15 \mathrm{~m}<\mathrm{x}<2.20 \mathrm{~m})$ are caused by the poloidal periodic Faraday screen bars. Except this, the wave fields in the near SOL, pedestal and further inside are quite smooth. At the time slice during the violent ELM crash ( $\mathrm{t}=25.25 \mathrm{~ms}$ ), prominent density filaments develop both in the pedestal and SOL. The density is poloidally rather inhomogeneous, with a typical density perturbation in the level of $80 \%$. As a result, the electric fields are strongly perturbed. Due to the mutual influence of multiple ELM filaments, poloidal stripe structures with poloidal extension of $\sim 0.1 \mathrm{~m}$ develop in the SOL, leading to regions of enhanced and reduced power flow. It should be noted that a single filament will induce a global perturbation of wave fields, and a local perturbation of wave fields is caused by the mutual influence of multiple filaments. But roughly 
speaking, the regions of increased SOL density are correlated with the regions of reduced wave fields, indicating the local filaments play a more important role than the remote filaments in comparison. In addition, the stripe structures starting from the SOL can extend further inside, even if there is no density perturbation inside the pedestal. This suggests that this scattering effect is a global effect.

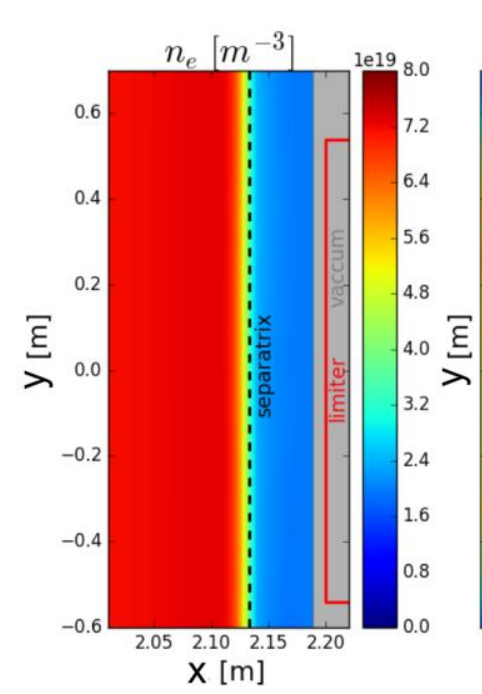

Before ELM $\left(\mathrm{t}_{1}=22.855 \mathrm{~ms}\right)$
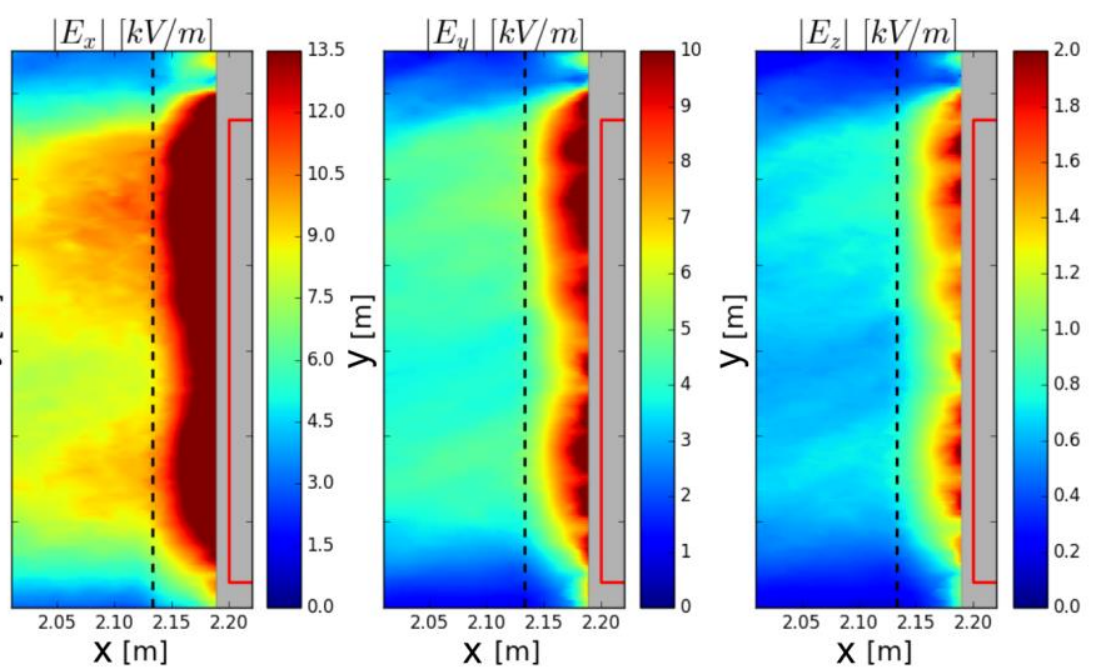

During ELM $\left(\mathrm{t}_{7}=25.255 \mathrm{~ms}\right)$
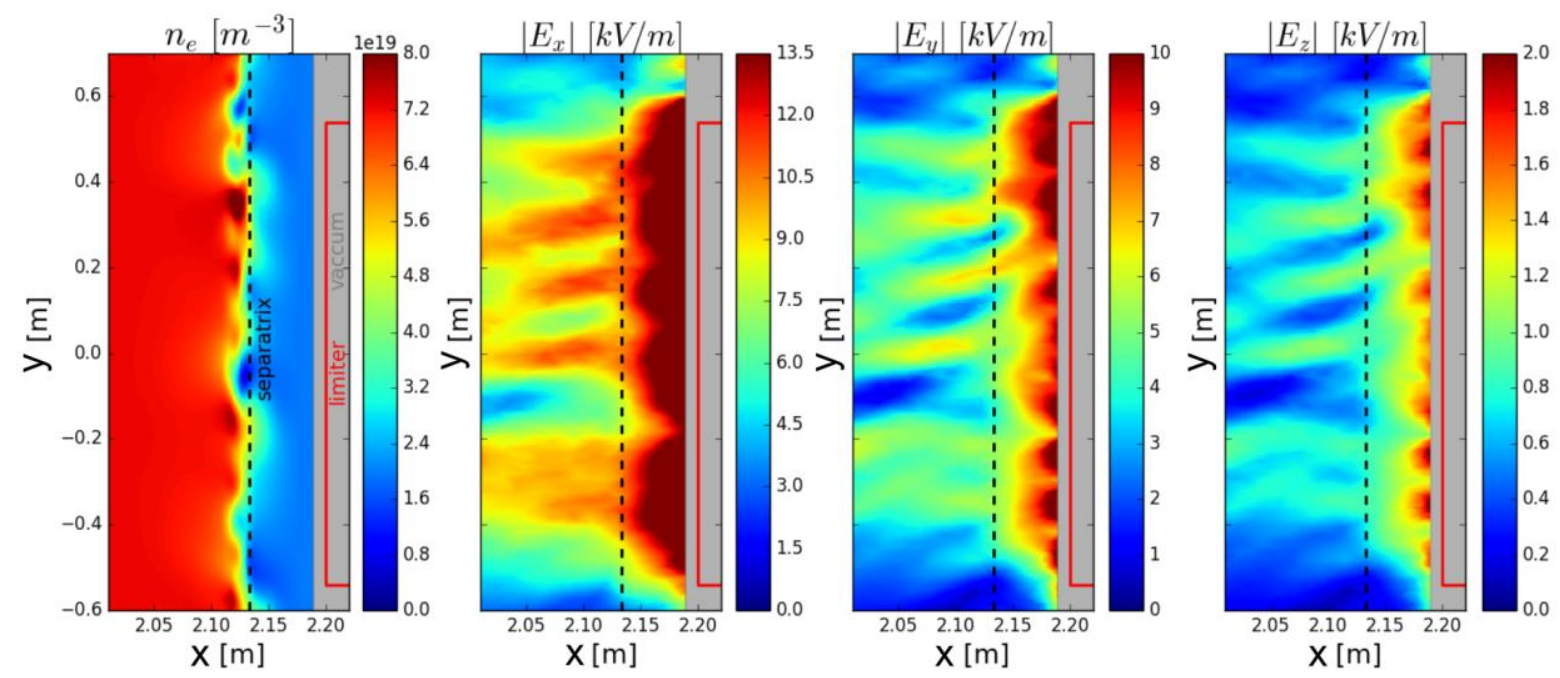

Figure 12. Comparisons of the electron density, $x, y$ and $z$ components of the electric field at time slices before ( $t=22.85 \mathrm{~ms}$ ) and during ( $\mathrm{t}=25.25 \mathrm{~ms}$ ) the ELM for the high boundary density case. The toroidal location of these poloidal cross-sections is $z=0.2 \mathrm{~m}$. 
(a) $\left|E_{x}\right|[\mathrm{kV} / \mathrm{m}]$
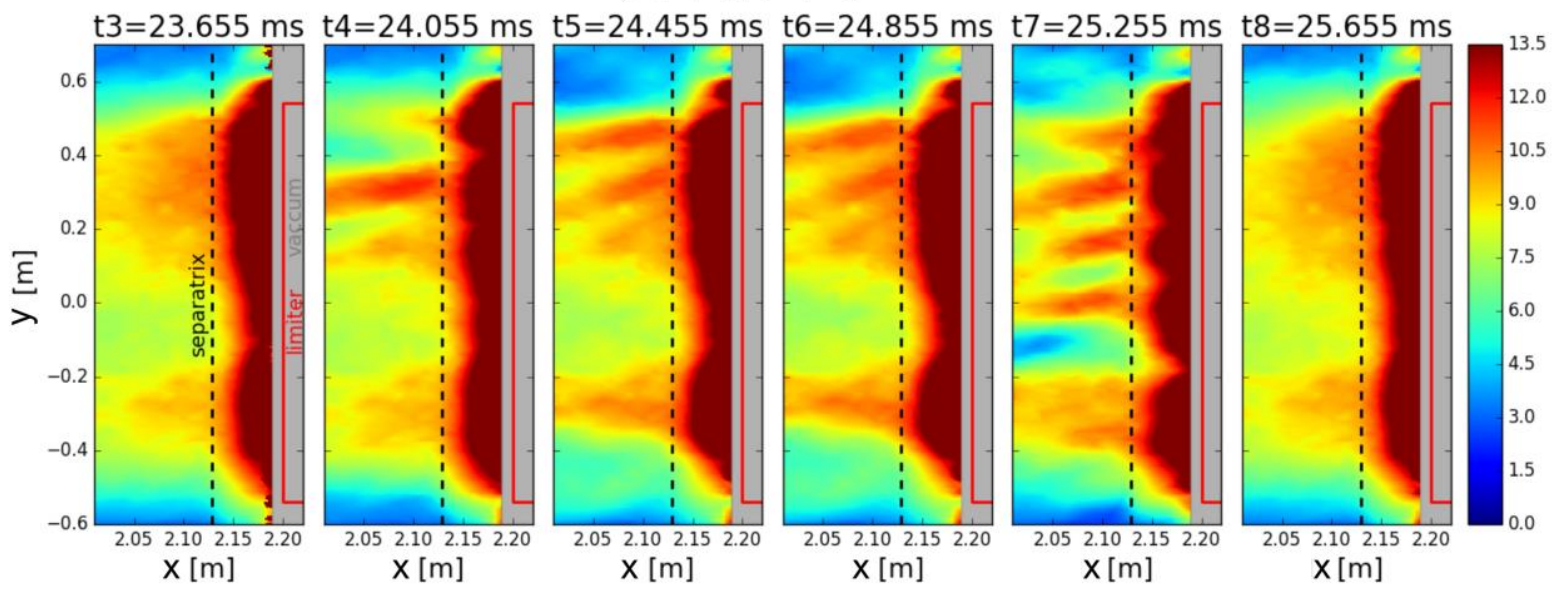

(b) $P_{x}\left[M W / m^{2}\right]$
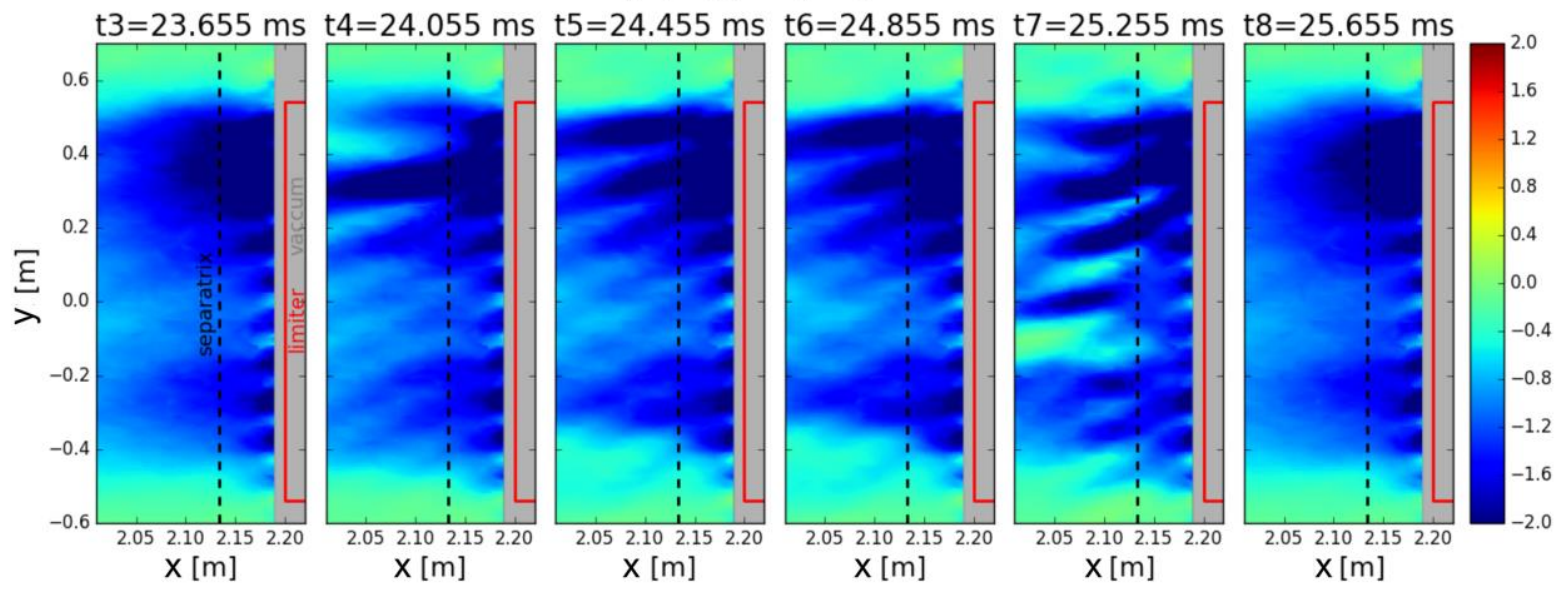

Figure 13. (a) The evolution of the magnitude of electric field during an ELM. (b) The evolution of the radial Poynting flux during an ELM.

Moreover, the evolution of the radial electric field and Poynting flux during an ELM is shown in Fig. 13. Both of them are strongly perturbed and become rather inhomogeneous in the poloidal direction. Clear stripe structures develop when the ELM or the magnetic energy of MHD modes becomes significant, for instance at time slices from t4 to t7. In the following, we will use $\delta n_{e}=\left(n_{e, E L M}-n_{e, 0}\right) / n_{e, 0}, \delta E_{i}=\left(E_{i, E L M}-E_{i, 0}\right) / E_{i, 0}$ and $\delta P_{i}=\left(P_{i, E L M}-P_{i, 0}\right) / P_{i, 0}$ to represent the perturbation of density, electric field and Poynting flux, respectively. Here, $n_{e, 0}, E_{i, 0}$ and $P_{i, 0}$ are the parameters at a time slice before the ELM (t=t1) and are used as references. $i$ is the $x, y$ or $z$ component of the wave field. At the peak of ELM (t=t7), $\delta n_{e} \sim 80 \%$, leading to $\delta E_{x} \sim 40 \%, \delta E_{y} \sim 80 \%$ and $\delta E_{z} \sim 80 \%$. These perturbations are smaller at other time slices. For instance, at time slice just before the ELM peak (t=t6), $\delta n_{e} \sim 60 \%, \delta E_{x} \sim 25 \%, \delta E_{y} \sim 50 \%$ and $\delta E_{z} \sim 50 \%$. The induced perturbation of the radial Poynting flux $\left(P_{x}\right)$ at the ELM peak ( $\left.\mathrm{t}=\mathrm{t} 7\right)$ is roughly $\sim 100 \%$. The development of stripe structures of the Poynting flux makes the energy carried by the wave rather poloidally inhomogeneous.

The wave heating in the plasma core can be affected if the Poynting flux reaching the resonance layer in the plasma core is poloidally inhomogeneous. To address this issue, the RAPLICASOL simulation region is extended 
radially to the Hydrogen minority resonance layer $(R=1.78 \mathrm{~m})$ while the ELM density $\left(t_{7}=25.255 \mathrm{~ms}\right)$ as well as other settings are kept the same. Although the core density is not perturbed, the poloidal inhomogeneity of the wave fields due to wave scattering in the SOL are able to reach the resonance layer (Fig. 14), a location $\sim 0.42 \mathrm{~m}$ away from the antenna. In particular, the stripe structures of the wave fields developed in the SOL can radially extend to the resonance layer and cause much of the poloidal inhomogeneity. Similarly, simulations with circular filaments and plane wave in section 2 also indicate that the poloidally inhomogeneous Poynting flux can travel $\sim 0.5 \mathrm{~m}$ away from the filament in the radial direction. Thus, the ICRF wave scattering is a quite global effect even though the scattering source is local. It is expected that this poloidally inhomogeneous wave fields and Poynting flux at the resonance layer will lead to poloidally inhomogeneous heating and change the radial heat deposition profile.

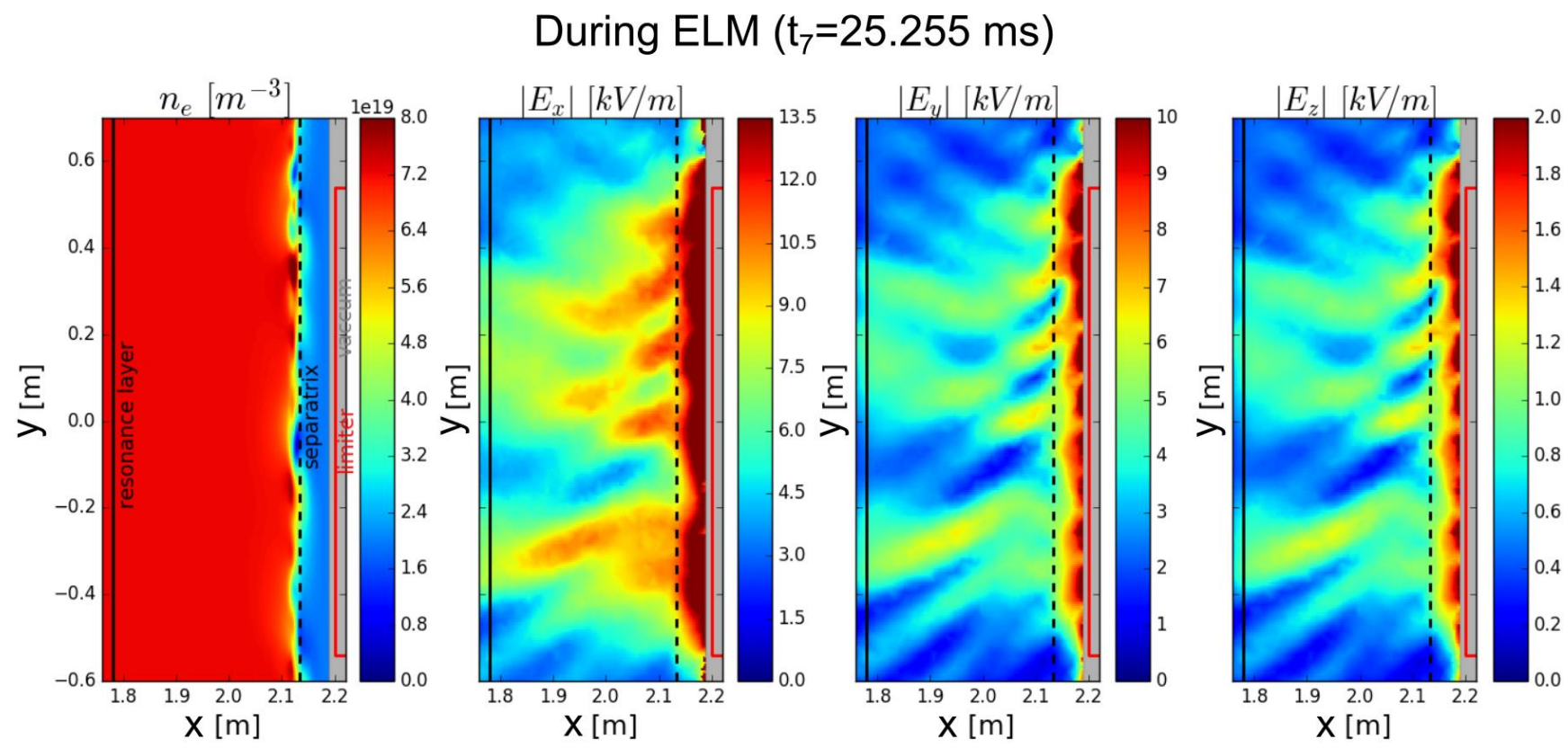

Figure 14. Simulations with the plasma region radially extended to the Hydrogen minority resonance layer $(R=1.78 m)$ for the ELM case. The ELM density is the same as the one in Fig. 12.

For plasma heating in the core, it is the perpendicular (perpendicular to B) component of the electric field which plays a crucial role. The parallel component of the electric field is not important for core heating, but can be important for plasma-wall interactions in the plasma edge, since it is usually considered as the source of enhanced RF-sheath potential and RF-specific impurity sputtering. It can be calculated by $E_{\|}=E_{z} \cos \theta+$ $E_{y} \sin \theta$, in which $\theta=9^{\circ}$ is the tilt angle between the toroidal direction (i.e. z-axis) and the magnetic field line direction. RAPLICASOL includes this tilt angle of the magnetic field in its formulation of the cold plasma dielectric tensor. Since $\delta E_{y} \sim \delta E_{z}$, the perturbation of $E_{||}$is in the same level as $\delta E_{y}$ or $\delta E_{z}$. The perturbation of $E_{||}$caused by the time-varying density profiles will likely lead to a modification of the RF rectified sheath potential. It is speculated that this is the reason for the observed discrepancies between measurements and simulated $E_{||}$with constant density profiles in the previous studies [23].

Furthermore, the radial Poynting flux $\left(P_{x}\right)$ on the innermost poloidal-toroidal cross-section of the simulation region (at $\mathrm{x}=2.0 \mathrm{~m}$ ) is analyzed (Fig. 15 ). It is shown that $P_{x}$ has a rather strong poloidal inhomogeneity during 
ELMs. The Poynting flux is enhanced at locations inside the stripe structures but is decreased in regions between the stripe structures. The largest $\left|P_{x}\right|$ value increases from $\sim 1.2 \mathrm{MW} / \mathrm{m}^{2}$ in the case before ELM to $\sim 1.9 \mathrm{MW} /$ $\mathrm{m}^{2}$ in the case during ELM. In addition, the stripe structures of the Poynting flux not only have a radial elongation, but are also elongated along the magnetic field lines, though with very limited toroidal extension $(\sim 1.2-1.8 \mathrm{~m})$. This further confirms that the RF power reaching the plasma core can be very poloidal inhomogeneous. It is likely that the ICRF heating in the plasma core are rather strong at some poloidal locations but very weak at other poloidal locations during ELMs.
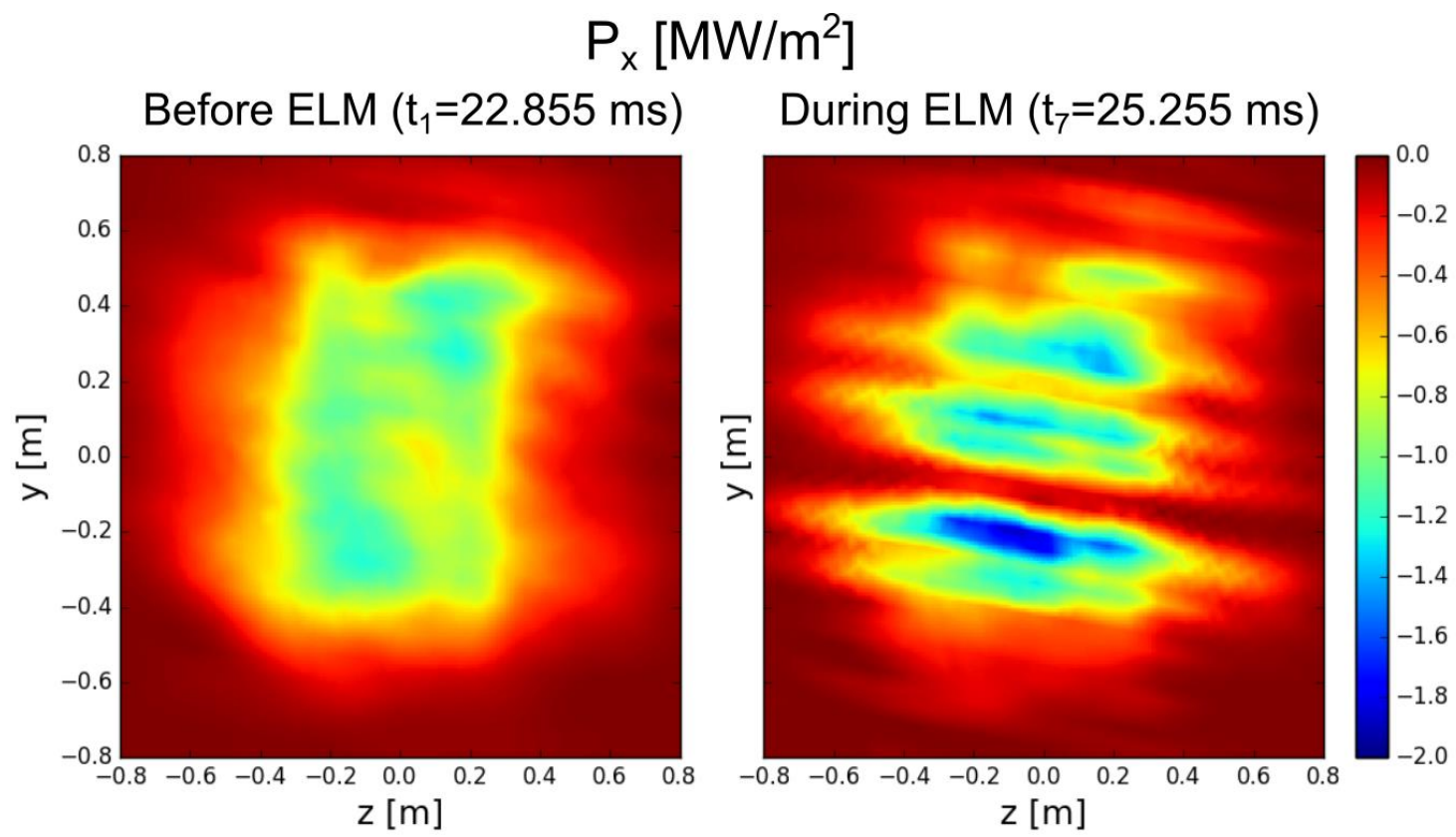

Figure 15. The radial Poynting flux from the antenna to the core on the $y-z$ plane (poloidal-toroidal cross-section) at $x=2.0 \mathrm{~m}$ (separatrix location at $\mathrm{x}=2.138 \mathrm{~m}$ and cyclotron resonance layer at $1.78 \mathrm{~m}$ ).

\subsection{Simulations with a low boundary density}

In this section, the case with a lower far SOL density $\left(2.1 \times 10^{18} \mathrm{~m}^{-3}\right)$ is studied, in the hope that by using a boundary density lower than the fast wave cut-off density $\left(4 \times 10^{18} \mathrm{~m}^{-3}\right)$, the modifications of the fast wave evanescent layer and thus the corresponding changes of the ICRF power coupling can be taken into account. However, the simulated density profiles (Fig. 16) show that the width of evanescent layer is only slightly varied, even at the peak of ELM ( $t=t 9)$. The most significant feature is still the big collapse of the pedestal density and the increase of the SOL density. Consequently, the main contribution to the perturbation of wave fields and antenna coupling resistance still comes from the density perturbation in the pedestal and SOL. The largest contribution to the magnetic energy of the MHD modes is the $n=2$ mode in the low boundary density case, in contrast to the $n=4$ mode in the high boundary density case. In addition, the ELM crash is larger for the low boundary density case. 

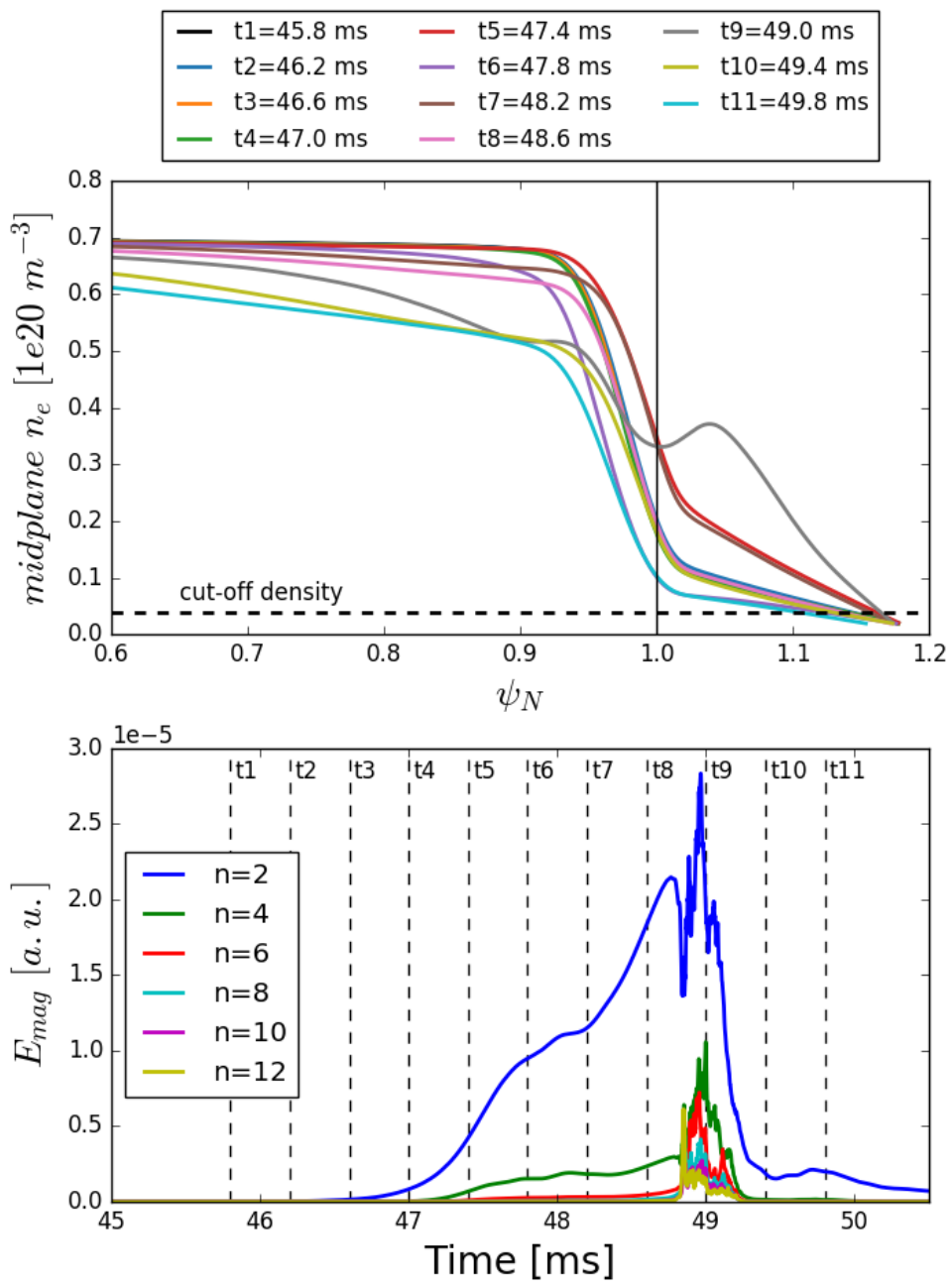

Figure 16. (a) The outer midplane density profiles with a low fixed boundary density. (b) The magnetic energy of the non-axisymmetric perturbations during an ELM.

Since the density perturbation during an ELM in the low boundary density case is larger than the high boundary density case, the perturbation of wave fields is also larger. The density and electric fields at time slices before and during an ELM for the low boundary density case are shown in Fig. 17. At the peak of ELM $\left(\mathrm{t}=\mathrm{t}_{9}\right), \delta n_{e}$ can be as large as $200 \%$, especially at regions near the separatrix. This leads to $\delta E_{x} \sim 100 \%, \delta E_{y} \sim 160 \%$ and $\delta E_{z} \sim 160 \%$. As a result, the radial Poyting flux is also significantly perturbed, and this perturbation on average is in the level of $200 \%$. Clear stripe structures are also developed and extend from the SOL towards the plasma core. 


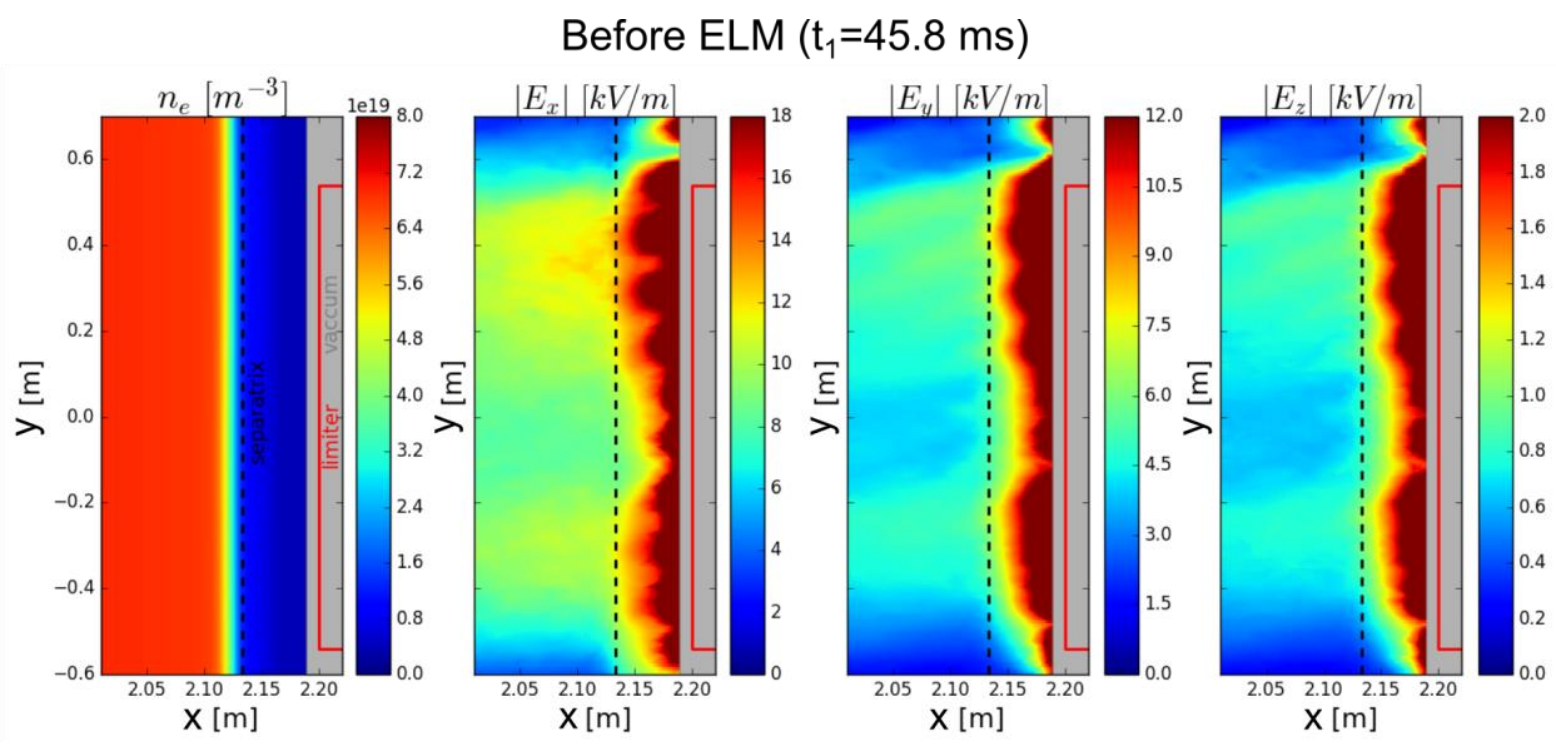

During ELM $\left(\mathrm{t}_{9}=49.0 \mathrm{~ms}\right)$

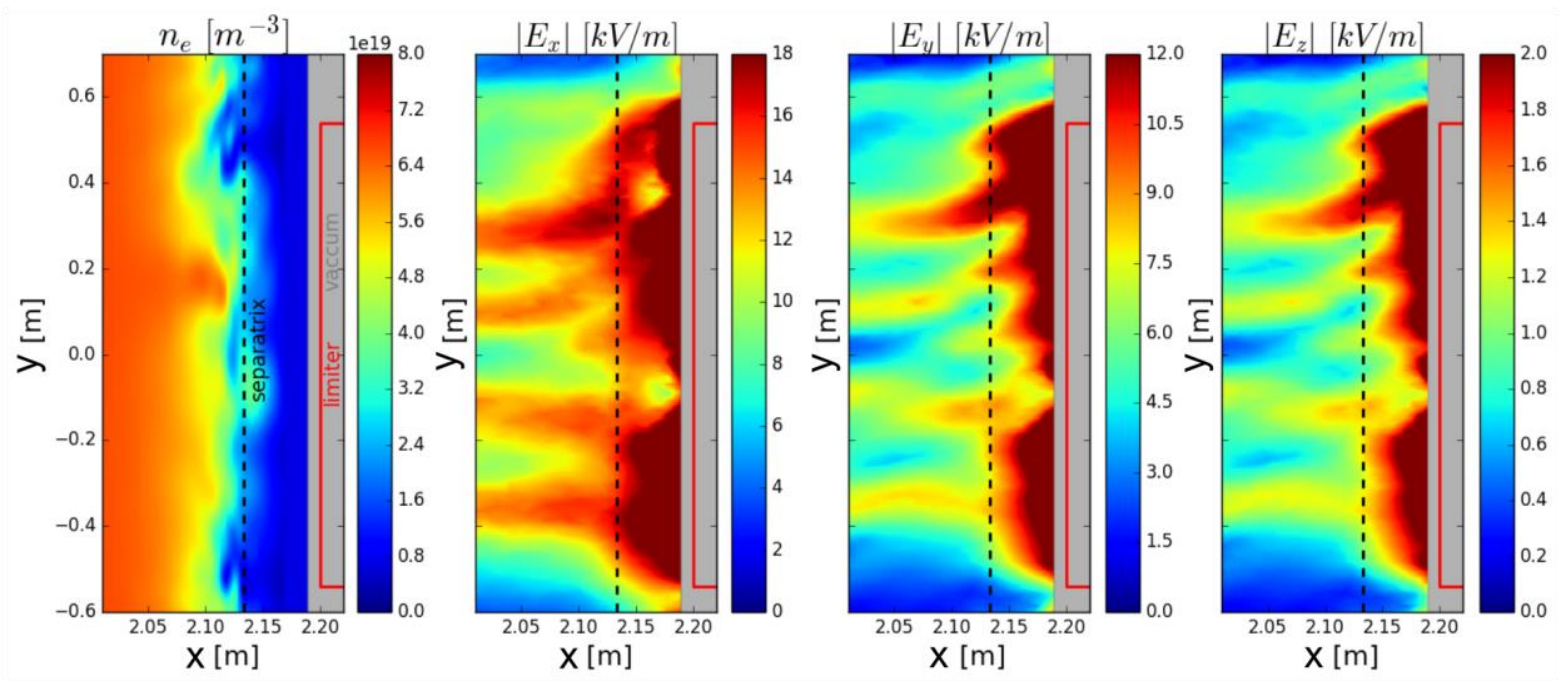

Figure 17. Comparisons of the electron density, as well as the $x$ and $y$ components of the electric field at the time slices before $\left(t_{1}=45.8 \mathrm{~ms}\right)$ and during $\left(t_{9}=49.0 \mathrm{~ms}\right)$ the ELM for the low boundary density case. The toroidal location of these poloidal cross-sections is $\mathrm{z}=0.2 \mathrm{~m}$.

The small change of fast wave evanescent layer in the low boundary density case has little influence on the calculated coupling resistance. In fact, the large collapse of density, especially at the peak of ELM, can significantly decrease the gradient of density profile at the pedestal and couple more power into the plasma. The RAPLICASOL calculations show that the coupling resistance is $\sim 3.0 \Omega$ at the peak of ELM $\left(\mathrm{t}=\mathrm{t}_{9}\right)$ while it is only about $1.6 \Omega$ before the ELM $\left(t=t_{1}\right)$. The calculated coupling resistance for the high boundary density case however remains roughly the same during the whole ELM. This is because in this case the density gradient at the pedestal is not significantly changed during an ELM. The loss of stored plasma energy during the ELM is about $25 \mathrm{~kJ}$ in the high boundary density case and about $80 \mathrm{~kJ}$ in the low boundary density case. The larger ELM crash in the latter case eventually leads to much larger coupling resistance modifications. This is consistent with the 
previous finding in AUG [24], which shows that the coupling resistance depends almost linearly on the loss of stored plasma energy.

\section{$\mathrm{P}_{\mathrm{x}}\left[\mathrm{MW} / \mathrm{m}^{2}\right]$}
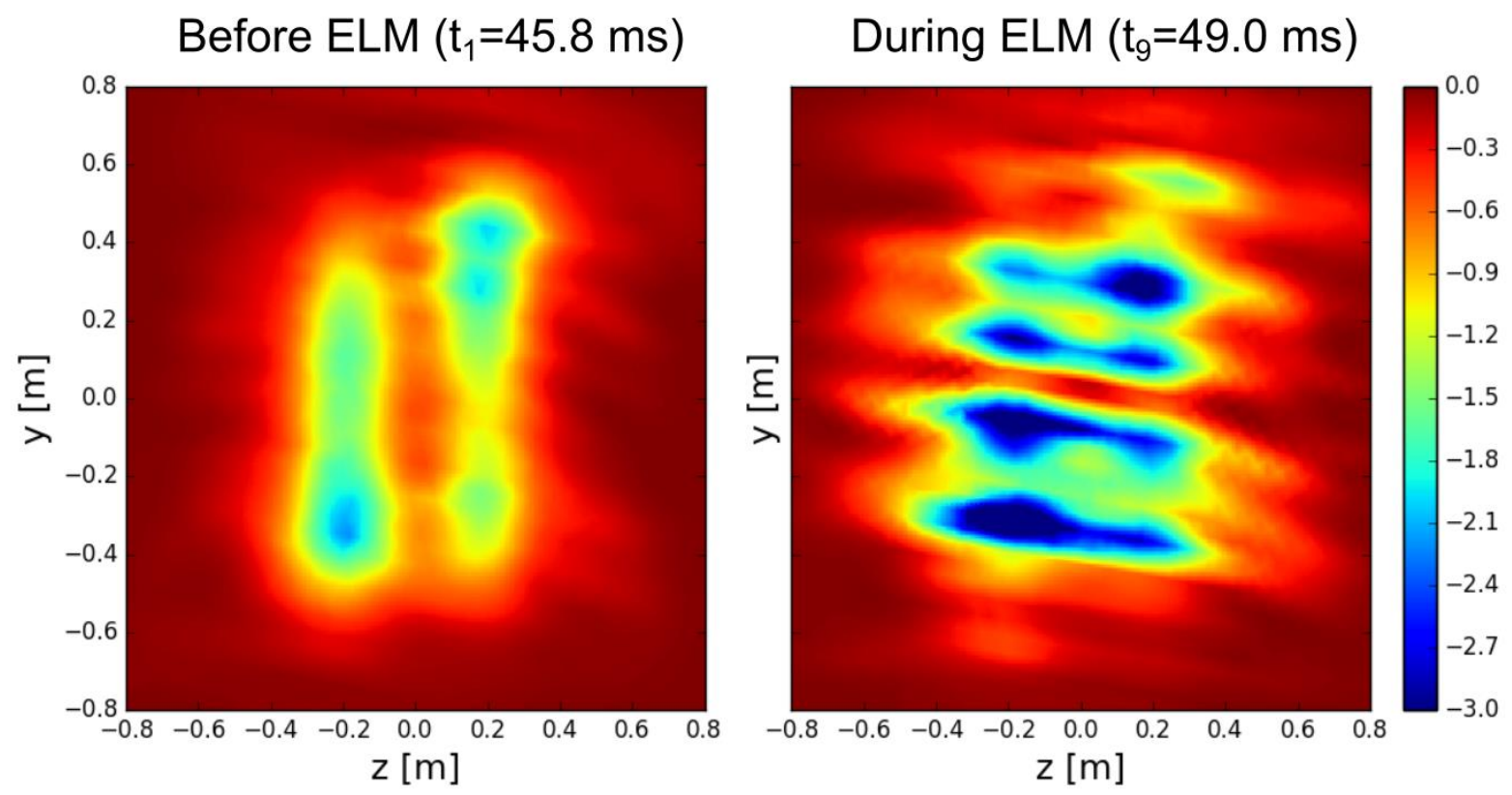

Figure. 18 The radial Poynting flux from the antenna to the core on the $y-z$ plane (poloidal-toroidal cross-section) at $x=2.0 \mathrm{~m}$ (separatrix location at $\mathrm{x}=2.138 \mathrm{~m}$ ).

Finally, the radial Poynting flux from the antenna to the core is analyzed (Fig. 18). Again, $P_{x}$ shows a strong poloidal inhomogeneity during ELMs. Because the power coupled to the plasma in the case during the ELM is higher by $\sim 63 \%$ than that before the ELM, the total power reaching the radial simulation boundary is also higher in the former case. The largest $\left|P_{x}\right|$ value increases from $\sim 2.2 \mathrm{MW} / \mathrm{m}^{2}$ before the ELM to $\sim 4.1 \mathrm{MW} / \mathrm{m}^{2}$ during the ELM. Compared to the high boundary density case, the perturbation of $\left|P_{x}\right|$ during ELM in the low boundary density case is stronger. This will lead to a more inhomogeneous heating in the plasma core.

\section{Conclusion}

The scattering of ion cyclotron range of frequency waves by filaments and ELMs has been comprehensively studied. Firstly, a 2D COMSOL model calculating the wave equation is developed to understand the influence of filaments on RF wave scattering. It is shown that a single density filament can lead to a global perturbation of wave fields. The largest perturbation of wave fields usually locates inside or near the filament. A scattering cone, starting from the center of the filament, often occurs in the direction of wave propagation. The standard deviation of radial Poynting flux, which is used to indicate the level of scattering, depends roughly linearly on the filament radius and filament density before it gets saturated at $\delta n_{e} \geq 1$ or $2 r_{F} / \lambda \geq 0.4$. The wave scattering caused by multiple density filaments is more complicated. The perturbation of wave fields is global and the maximum values are not necessary located near the filament. The scattering cone becomes less obvious as the 
number of filament or the distance between filaments increases. Instead, poloidal distributed and radially elongated stripe structures with enhanced and reduced electric fields develop. The standard deviation of radial Poynting flux depends roughly linearly on the number of filaments, but nonlinearly on the distance between filaments.

More realistic simulations by coupling the JOREK and RAPLICASOL codes are then performed to understand the influence of ELMs on RF wave scattering. The non-linear MHD code JOREK is used to calculate the 3D core and SOL density during type-I ELMs. The antenna code RAPLICASOL is then used to calculate the electric fields and Poynting flux. Two cases, one with a low far SOL density and one with a high far SOL density, have been investigated. The ELM crash and the density perturbations induced in the former case are much larger than those in the latter case. If considering results of both cases, ELMs can induce a density perturbation of the order of $80 \%-200 \%$ at the ELM peak. This will leads to a global perturbation of electric field of the order of $40 \%-160 \%$ and a global perturbation of Poynting flux of the order of $100 \%-200 \%$. Interestingly, due to the mutual influence of multiple density filaments and density holes, radially elongated but poloidally distributed stripe structures with enhanced and reduced wave fields develop. This finding is consistent with the 2D COMSOL simulation results when multiple filaments are taken into account. In addition, the stripe structures originating from the SOL and can extend radially into the core plasma. It is expected that due to this mechanism, the RF heating in the resonance layer will be poloidally inhomogeneous, and the radial heat deposition profile will be affected.

In the paper, we mainly studied the scattering of RF wave by filaments and ELMs. In fact, as shown by the experimental results, ELMs can also influence the antenna power and ICRF coupling significantly as the SOL density is changed. On the one hand, more power is reflected in the transmission line and less power is emitted by the antenna, as the impedance matching condition is changed due to the modification of SOL density profiles during ELMs. On the other hand, the antenna loading resistance becomes larger, since the increase of SOL density leads to a decrease of the width of the evanescent layer. A combination of the two competing effects leads to a decrease of coupled power from the antenna to the plasma during ELMs.

In the future, we attempt to investigate the influence of RF wave scattering on the RF power absorption in the plasma core by using the 3D ELM density calculated by JOREK in the 3D HIS-TORIC code [25]. The later code is able to calculate the propagation of the wave from the antenna and the absorption of RF waves in the plasma core.

\section{ACKNOWLEDGMENTS}

This work has been carried out within the framework of the EUROfusion Consortium and has received funding from the Euratom research and training programme 2014-2018 and 2019-2020 under grant agreement No 633053. The views and opinions expressed herein do not necessarily reflect those of the European Commission. Some of the simulations were performed using the MARCONI-FUSION supercomputer. 


\section{References}

[1] Stix T. H. 1992 Waves in Plasmas American Inst. of Physics

[2] Schubert M. et al 2015 18th Joint Workshop on Electron Cyclotron Emission and Electron Cyclotron Resonance Heating 8702010

[3] Myra J. R. et al 1994 Physics of Plasmas $12890-900$

[4] Ram A. K. et al 2013 Physics of Plasmas 20056110

[5] Ram A. K. et al 2016 Physics of Plasmas 23022504

[6] Myra J. R. et al 2010 Physics of Plasmas 17102510

[7] loannidis Z. C. et al 2017 Physics of Plasmas 24102115

[8] Snicker A. et al 2018 Nuclear Fusion 58016002

[9] Valvis S. I. et al 2018 Journal of Plasma Physics 84745840604

[10] Huysmans G. T. A. et al 2007 Nuclear Fusion 47 659-66

[11] Czarny O. et al 2008 Journal of Computational Physics 227 7423-45

[12] Jacquot J. et al 2013 Plasma Physics and Controlled Fusion 55115004

[13] Tierens W. et al 2019 Nuclear Fusion 59046001

[14] Mie G. 1908 Annalen der Physik 330 377-445

[15] Lockwood D. J. 2016 Rayleigh and Mie Scattering New York, NY Springer New York

[16] D'Ippolito D. A. et al 2002 Physics of Plasmas 9 222-33

[17] Snyder P. B. et al 2002 Physics of Plasmas 9 2037-43

[18] Snyder P. B. et al 2004 Nuclear Fusion 44 320-8

[19] Franck E. et al 2015 ESAIM: M2AN 49 1331-65

[20] Orain F. et al 2013 Physics of Plasmas 20102510

[21] Cathey Cevallos A. et al 2020 To be submitted to Physical Review Letters

[22] Mink A. F. et al 2018 Nuclear Fusion 58026011

[23] Bobkov V. et al 2017 Plasma Phys. Control. Fusion 59014022

[24] Bobkov V. V. et al 2006 Nuclear Fusion 46 S469-S75

[25] Shiraiwa S. et al 2017 Nuclear Fusion 57086048 